\title{
A non-coding function of TYRP1 mRNA promotes melanoma growth
}

\author{
David Gilot ${ }^{1,11,12}$, Mélodie Migault ${ }^{1,11}$, Laura Bachelot ${ }^{1}$, Fabrice Journé ${ }^{2}$, Aljosja Rogiers ${ }^{3,4}$, \\ Emmanuelle Donnou-Fournet ${ }^{1}$, Ariane Mogha ${ }^{1}$, Nicolas Mouchet ${ }^{1}$, Marie-Laure Pinel-Marie ${ }^{5}$, Bernard Mari ${ }^{6}$, \\ Tristan Montier ${ }^{7,8}$, Sébastien Corre ${ }^{1}$, Arthur Gautron ${ }^{1}$, Florian Rambow ${ }^{3,4}$, Petra El Hajj ${ }^{2}$, Rania Ben Jouira \\ Sophie Tartare-Deckert ${ }^{9}$, Jean-Christophe Marine ${ }^{3,4}$, Brice Felden ${ }^{5}$, Ghanem Ghanem ${ }^{2}$ \\ and Marie-Dominique Galibert ${ }^{1,10,12}$
}

${ }^{1}$ CNRS UMR 6290, IGDR, Université de Rennes I, 35043 Rennes, France. ${ }^{2}$ Institut Jules Bordet, Université Libre de Bruxelles, 1000 Brussels, Belgium. ${ }^{3}$ Department of Oncology, KU Leuven, 3000 Leuven, Belgium. ${ }^{4}$ VIB Center for Cancer Biology, VIB, 3000 Leuven, Belgium. ${ }^{5}$ Inserm U1230, Université de Rennes I,

35043 Rennes, France. ${ }^{6} \mathrm{CNRS}$ UMR 7275, 06560 Sophia Antipolis, France. ${ }^{7}$ Inserm U1078, Université de Bretagne Occidentale, 29218 Brest, France. ${ }^{8} \mathrm{CHRU}$ de Brest, Laboratoire de Génétique Moléculaire et d'Histocompatibilité, 29200 Brest, France. ${ }^{9}$ Inserm U1065, Equipe labellisée Ligue contre le Cancer 2016, C3M,

06204 Nice, France. ${ }^{10} \mathrm{CHU}$ Rennes, Génétique Somatique des Cancers, 35033 Rennes, France. ${ }^{11}$ These authors contributed equally to this work.

${ }^{12}$ Correspondence should be addressed to D.G. or M.-D.G. (e-mail: david.gilot@univ-rennes1.fr or mgaliber@univ-rennes1.fr)

Competition among RNAs to bind miRNA is proposed to influence biological systems. However, the role of this competition in disease onset is unclear. Here, we report that TYRP1 mRNA, in addition to encoding tyrosinase-related protein 1 (TYRP1), indirectly promotes cell proliferation by sequestering miR-16 on non-canonical miRNA response elements. Consequently, the sequestered miR-16 is no longer able to repress its mRNA targets, such as RAB17, which is involved in melanoma cell proliferation and tumour growth. Restoration of miR-16 tumour-suppressor function can be achieved in vitro by silencing TYRP1 or increasing miR-16 expression. Importantly, TYRP1-dependent miR-16 sequestration can also be overcome in vivo by using small oligonucleotides that mask miR-16-binding sites on TYRP1 mRNA. Together, our findings assign a pathogenic non-coding function to TYRP1 mRNA and highlight miRNA displacement as a promising targeted therapeutic approach for melanoma.

MicroRNAs (miRNAs) are short ( $\sim 22$ nucleotides) non-coding RNAs that play critical roles in many biological processes and diseases, including oncogenesis ${ }^{1}$. miRNAs guide the silencing Argonaute protein complex to messenger RNA targets to trigger post-transcriptional repression. Perfect base pairing between miRNA seed sequences (nucleotides 2 to 7) and miRNA response elements (MREs) of an mRNA inhibits its translation and mediates mRNA decay ${ }^{2}$. Imperfect seed pairings are also functional, although Argonaute interaction and repression are weaker ${ }^{3-5}$. In addition to these miRNA-mRNA interactions leading to post-transcriptional repression, independent studies and approaches have identified a large number of non-canonical binding sites, referred to here as non-canonical MREs ${ }^{3,5-9}$. Two recent meta-analyses confirmed that non-canonical MREs efficiently interact with miRNAs ${ }^{4,5}$. While the first study considered these non-canonical binding sites as 'non-functional' because they do not mediate mRNA decay and show no sign of sequence conservation ${ }^{4}$, the second study advocated for biologically relevant roles because they identified a few different types of non-canonical binding site that could downregulate
mRNA expression ${ }^{5}$. Considering the diversity of the nature and function of non-canonical MREs, it is accepted that miRNAs may control gene regulatory networks in a far more complex manner than is currently understood.

One of the common approaches developed to study the biological function of specific miRNAs in vitro and in vivo makes use of artificial exogenous DNA constructs, called miRNA sponges. These contain multiple binding sites for the miRNA of interest, thereby specifically inhibiting a given miRNA and de-repressing its RNA targets ${ }^{10-13}$. miRNA sponges are thus potent miRNA inhibitors. Recently, some endogenous miRNA sponges have been described, including circular RNAs, among others ${ }^{14-19}$. Similarly, circular RNAs contain dozens of canonical MREs for a single miRNA species and show resistance against miRNA-mediated RNA decay. Their circular shape is thought to support this latter property ${ }^{20-23}$.

These observations raise the interesting possibility that competition among RNAs to sequester miRNAs may have important physiological and pathological functions ${ }^{18,24}$. However, the exact 
number of players in these competitions, including natural miRNA sponges and non-canonical MREs, remains largely unknown due to the lack of dedicated predictive algorithms.

We recently demonstrated that elevated mRNA expression of tyrosinase-related protein $1(T Y R P 1)^{25,26}$ in metastatic melanoma biopsies correlates with poor overall patient survival ${ }^{27-29}$. Additionally, we and others have shown that the single-nucleotide polymorphism (SNP) rs683, located in one MRE-155 on the $3^{\prime}$ untranslated region ( $3^{\prime}$ UTR) of TYRP1, strongly reduces miR-155-induced decay ${ }^{27,30}$, thereby contributing to elevated levels of TYRP1 mRNA. However, the molecular mechanisms linking TYRP1 mRNA to the survival of metastatic melanoma patients remain unknown.

In the current study, we establish that TYRP1 mRNA efficiently sequesters miR-16, cancelling the repressive activity of miR-16 on its targets, including $R A B 17$ mRNA. We demonstrate that RAB17 controls melanoma cell proliferation and tumour growth. Moreover, overall survival of metastatic melanoma patients can be predicted by estimating the level of active miR-16. Importantly, we developed a series of therapeutic strategies that efficiently overcome melanoma addiction to miR-16 sponge.

In this study, we uncover the mechanism by which TYRP1 mRNA can sequester miR-16, thereby governing a specific gene expression program that promotes melanoma growth. These data have broad implications both for our understanding of cancer biology and for the development of targeted therapeutics.

\section{RESULTS}

\section{TYRP1 mRNA drives melanoma growth}

Elevated mRNA levels of TYRP1 in metastatic melanoma biopsies correlate with poor clinical outcome of patients. Unexpectedly, the TYRP1 protein could not be detected in half of the tissues expressing TYRP1 mRNA ${ }^{27-29}$. We explored the molecular mechanism linking the level of TYRP1 mRNA, but not protein, to patient outcomes and showed that high levels of TYRP1 mRNA confer a growth advantage to metastatic melanoma cells (Fig. 1a and Supplementary Table 1). Knockdown (KD) of TYRP1 mRNA using small hairpin RNA (shRNA) compromised the proliferation of 501Mel, ME1402 and Mel624 melanoma cells, and of MM057 and MM165 short-term melanoma cells (Fig. 1b,c and Supplementary Fig. 1a-d). Similar results have been obtained using alternative silencing approaches with short interfering RNA (siRNA) ${ }^{31}$ and antisense-mediated $\mathrm{KD}^{32}$, thereby excluding off-target effects of TYRP1 shRNA (shTYRP1). Conversely, ectopic expression of TYRP1 mRNA significantly increased melanoma cell proliferation (Fig. 1d). To broaden the biological role of TYRP1 mRNA, we performed in vivo experiments. Silencing TYRP1 mRNA significantly reduced the tumour growth of engrafted SKMel28-luc cells in immunocompromised mice (Fig. 1e,f). Although SKMel28-luc cells express TYRP1 mRNA, we could not detect the TYRP1 protein in our conditions, as it was presumably under the detection limit of the three antibodies used (Fig. 1e and Supplementary Fig. 1e). These results, along with the results obtained with TYRP1-expressing 501Mel melanoma cells (Fig. 1b), suggest that high levels of TYRP1 mRNA promote cell proliferation and tumour growth irrespective of the level of TYRP1 protein. These data indicate that, in addition to its protein-coding function, TYRP1 mRNA may exert a non-coding biological function. We postulated that TYRP1 mRNA may act as a natural miRNA sponge to dampen the tumour-suppressor activity of miRNAs.

\section{TYRP1 mRNA sequesters miR-16}

To identify TYRP1-associated miRNAs, the $3^{\prime}$ UTR of TYRP1 was used as a bait in RNA immunoprecipitation experiments (Fig. 2a) ${ }^{33}$. Immunoprecipitated miRNAs were purified and quantified using a TaqMan low-density array (800 miRNAs). Two miRNAs (miR-155 and miR-16) were significantly enriched (Fig. 2b) independently of their expression levels. The highly expressed miR-211 was not co-purified, nor was miR-330, whose expression level was intermediate between those of miR-16 and miR-155 (Supplementary Table 2). In addition, miR-155 had already been shown to interact with the TYRP1 3' UTR, giving robustness to this approach ${ }^{30}$.

TargetScan, a computational method predicting miRNA targets ${ }^{4}$ according to perfect sequence match between an miRNA seed region and its targets, failed to detect MRE-16 on TYRP1. In contrast, the thermodynamics-based RNAhybrid algorithm, which takes into account energetically favourable hybridization of miRNA/target duplexes with secondary structures ${ }^{34}$, identified three putative MRE-16s (Fig. 2c) along with the three MRE-155s that were previously validated (Supplementary Fig. 2a,b). The identified MRE-16s did not exhibit the top-ranking GCUGCU motif described for canonical miR-16 targets $^{4,7,35}$, indicating the presence of non-canonical base pairings. Noncanonical MRE-16-binding sites are not predicted to mediate mRNA decay following miRNA binding ${ }^{4,5}$. Accordingly, in the presence of synthetic miR-16, the TYRP1 mRNA level was significantly increased (Fig. 2d) in contrast to what was observed with miR-155, which induced TYRP1 mRNA decay. Consistently, synthetic miR-16 increased the luciferase activity of chimaeric miRNA sensors containing the luciferase-coding sequence fused to TYRP1 non-canonical MRE-16 sequences. The increase was miR-16 sequence dependent (Fig. 2e).

Together, these results demonstrate that miR-16 does not induce TYRP1 mRNA decay despite its interaction with TYRP1 mRNA.

Given that miR-16 has been described as a tumour-suppressor miRNA ${ }^{36}$, we postulated that high expression levels of TYRP1 mRNA may sequester miR-16 to limit or negate its tumour-suppressor activity. Consistently, a significant decrease in cell density was observed following ectopic expression of synthetic miR-16 (Fig. 2f). These results are in agreement with the previously described anti-proliferative effect of miR-16 in melanoma cells ${ }^{37}$, and indicate that tumour-suppressor activity of miR-16 can be restored in cells expressing high levels of TYRP1 mRNA.

Our model predicts that a reduction in the TYRP1 mRNA level should restore the tumour-suppressor activity of miR-16. Accordingly, silencing TYRP1 caused a significant cell-density decrease. This effect was miR-16 dependent, being reversed by the transfection of a miR-16 inhibitor (anti-miR-16) (Fig. 2g). Together, our results support a model in which TYRP1 mRNA acts as an efficient miR-16 sponge to dampen miR-16 tumour-suppressor activity.

\section{Biological and clinical significance of TYRP1 mRNA as a miRNA sponge}

The efficacy of miRNA sponges in titrating specific miRNAs depends not only on the number and affinity of miRNA-binding sites but also on the abundance of the sponge relative to the level of the miRNAs to 
a

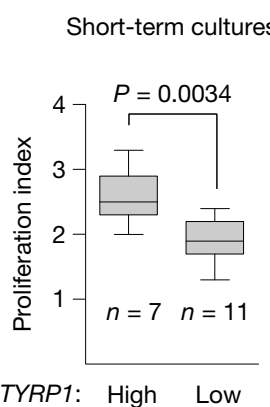

d

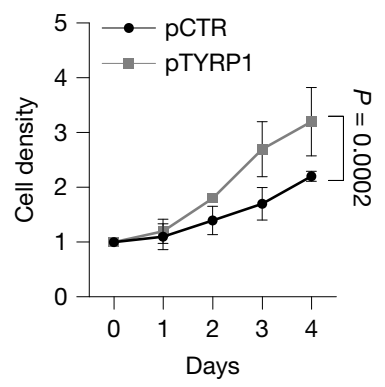

b
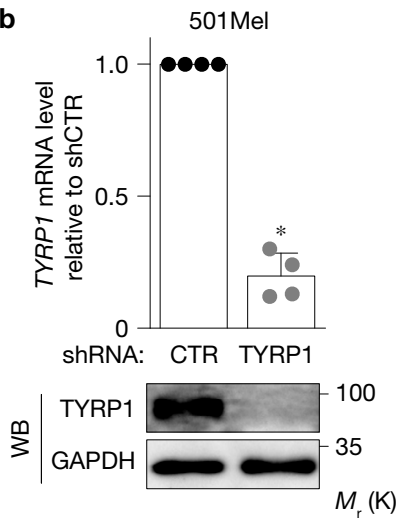

c

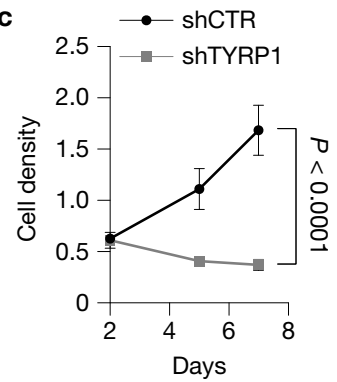

e

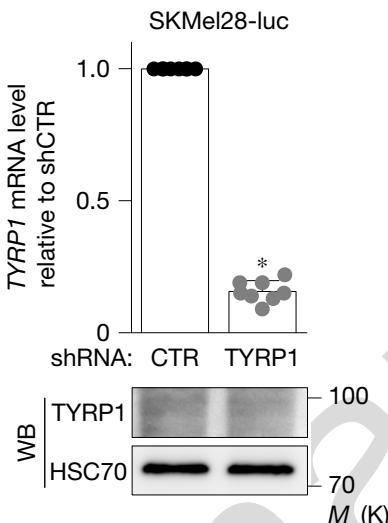

f

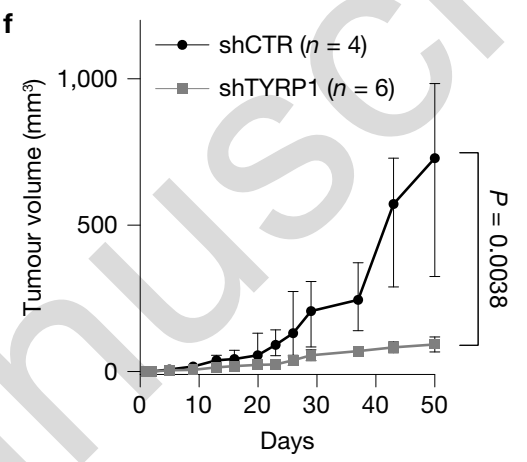

Figure 1 TYRP 1 mRNA drives melanoma growth. (a) Proliferative index of 18 short-term cultures established from melanoma biopsies (described in Supplementary Table 1). Box plots showing cell proliferation in groups defined according to their high ( $n=7$ independent short-term cultures) or low/absent ( $n=11$ independent short-term cultures) TYRP1 mRNA expression levels (with a cutoff $2-\Delta \mathrm{Ct}=0.1$ ); the boxes represent the median, and the first and third quartiles; whiskers represent the minimum and maximum of all data points. Two-sided Mann-Whitney test. (b) TYRPI knockdown (KD) in the $501 \mathrm{Mel}$ melanoma cell line expressing TYRP1 mRNA (top; $n=4$ biologically independent samples) and proteins (bottom, western blot). GAPDH serves as a loading control. (c) Proliferation rate of TYRP1 KD (shTYRP1) or control (shCTR) $501 \mathrm{Mel}$ cells ( $n=3$ biologically independent experiments). (d) Gain-of-function experiments using full-length TYRP1

be titrated ${ }^{38}$. We therefore quantified the number of TYRP1 mRNA and miR-16 molecules per cell by northern blotting. We detected approximately 3,300 molecules of TYRP1 mRNA and 4,800 copies of miR-16 per cell (Fig. 3a and Supplementary Fig. 2d,e). Given that TYRP1 mRNA displays three non-canonical MRE-16s per mRNA molecule, the entire pool of miR-16 can potentially be sequestered per cell.

The robustness of a sponge depends on its capacity to resist miRNA-mediated mRNA decay, indirectly allowing miRNA sequestration $^{13}$. We showed above that miR-16 does not induce TYRP1 mRNA decay (Fig. 2d,e). In addition, we and others have demonstrated that TYRP1 mRNA decay is under the control of miR-155 (refs 27,30) and that SNP rs683 alters one of the canonical MRE-155 sequences, reducing miR-155-dependent TYRP1 mRNA decay. While the $\mathrm{C}$ allele (TYRP1-C, SNP rs683) allows perfect seed-pairing and efficient miR-155-mediated decay of TYRP1 mRNA,
(pTYRP1) or a control (pCTR) in SKMel28-luc cells $(n=3$ biologically independent experiments). (e) TYRP1 KD in the SKMel28-luc melanoma cell lines: mRNA (top; $n=8$ biologically independent experiments) and protein levels (bottom, western blot). HSC70 serves as loading control. (f) Xenograft tumour volumes of shCTR and shTYRP1 SKMel28-luc cells ( $n=4$ and 6 mice respectively). Values correspond to the median and interquartile range. For $\mathbf{b}-\mathbf{e}$, values correspond to the mean \pm s.d. ${ }^{*} P<0.001$; $P$ values were calculated by two-sided unpaired $t$-tests with Welch's correction for $\mathbf{b}$ and $\mathbf{e}$; or by two-way ANOVA without adjustment for $\mathbf{c}, \mathbf{d}$ and f. Significant for $P<0.05$. Western blot results are representative of at least three independent experiments. Source data are available in Supplementary Table 8 and unprocessed original blots are shown in Supplementary Fig. 7.

the A allele (TYRP1-A, SNP rs683) reduces miR-155-dependent repression (Supplementary Fig. 2a-c).

Alone, synthetic miR-16 and miR-155 have opposite effects on TYRP1 decay. Thus, we performed competition assays to better characterize their combined activity on TYRP1 decay (Fig. 3b). At equimolar levels, synthetic miR-16 abrogated the TYRP1 mRNA decay induced by miR-155. It is important to note that, in melanoma biopsies, miR-16 is expressed at a higher level than miR-155 (Fig. 3c,d). These data suggest that endogenous miR-16 negates the effect of miR-155 on TYRP1-A. Thus, the level of TYRP1 expression and its related miRNA sponge activity are tightly associated with the SNP rs683 and the level of miR-16 in melanoma. Interestingly, we identified three non-canonical MRE-16s in the mouse TYRP1 3' UTR, one of which is in the vicinity of a canonical MRE-155 as observed for human TYRP1 (Supplementary Fig. 3a,b). TYRP1 KD and synthetic miR-16 efficiently reduced the proliferation of mouse melanoma cells (B16) 
a

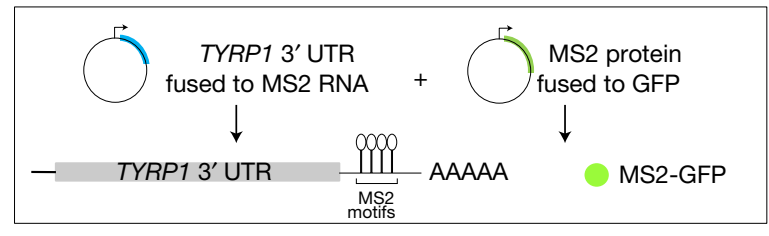

$\downarrow$ Transfection

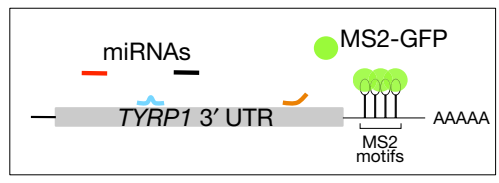

$\downarrow$ Immunoprecipitation

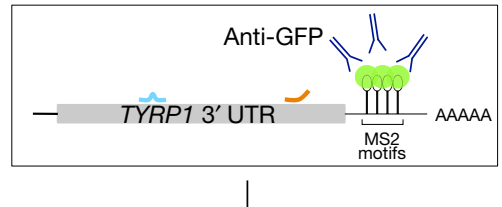

miRNA quantification $(n=800)$ d

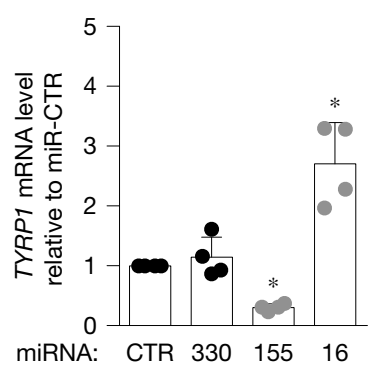

e

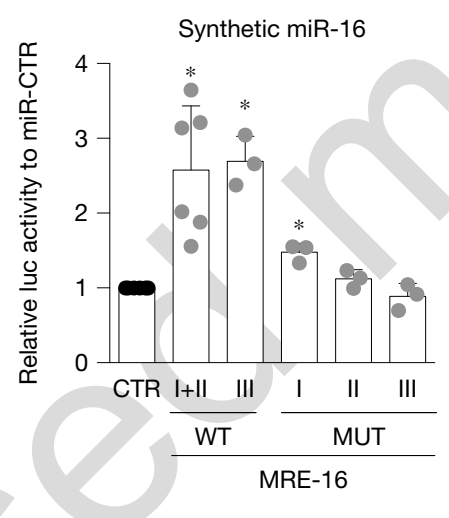

b

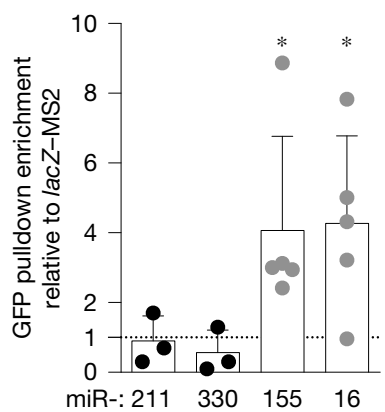

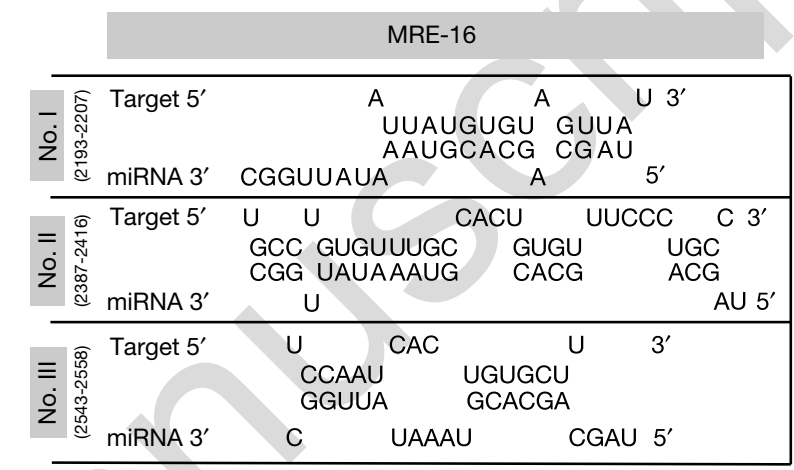

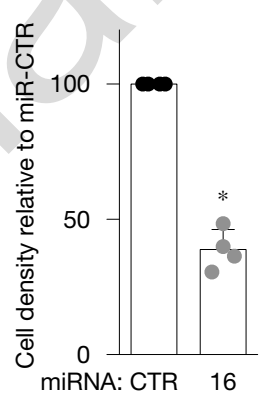

g

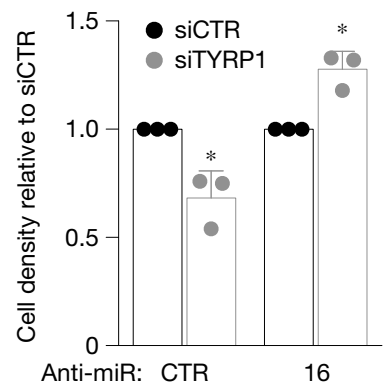

Figure 2 TYRP1 mRNA sequesters miR-16. (a) Schematic representation of MS2-RNA immunoprecipitation (MS2-RIP). The 3' UTR of TYRP1 was fused to MS2 RNA stem-loop motifs and co-transfected with the MS2GFP-expressing vector. The MS2-GFP protein is able to recognize the MS2 RNA motif. Anti-GFP antibody targeting the MS2-GFP protein was used to precipitate the miRNA/RNA complexes. (b) Quantification of isolated miRNA following TYRP1 $3^{\prime}$ UTR MS2-RIP. Values are normalized against the lacZ-MS2 enrichment $(n=3$ biologically independent experiments for miR-211 and miR-330 expression and $n=5$ biologically independent experiments for miR-155 and miR-16 expression); one-sided unpaired $t$-test with Welch's correction). (c) Three MRE-16s within the TYRP1 3' UTR sequence (NM_000550.1) were identified using RNAhybrid software ${ }^{34}$. (d) Expression levels of TYRP1 mRNA in $501 \mathrm{Mel}$ cells three days after transfection with either synthetic miR-16 or miR-155 or miR-330 or

(Supplementary Fig. 3c-e). Together, these results suggest that the TYRP1 sponge model may not be restricted to humans.

To determine whether TYRP1- $A$ ensured miR-16 sequestration, we knocked down TYRP1-A in a TYRP1-A homozygous cell line (501Mel) to release docked miRNAs from the sponge and used two miRNA sensors to measure the activity of released miR-155 and miR-16
miR-CTR ( $n=4$ biologically independent experiments; one-way ANOVA with Holm-Sidak's multiple comparisons test). (e) Effects of synthetic miR-16 on the identified regions of TYRP1 MRE-16 in 501Mel cells. MRE-16 no. I and no. II were present on the same luciferase reporter. MUT, mutated; WT, wild-type; $n=3$ and $n=6$ biologically independent experiments for WT I+II assay. (f) Cell density of $501 \mathrm{Mel}$ cells three days after transfection with miR-16 or miR-CTR ( $n=4$ biologically independent experiments). (g) Rescue experiments with anti-miR-CTR or anti-miR-16 on TYRP1 KD and control $501 \mathrm{Mel}$ cells. Cell density was evaluated three days after co-transfection (siRNA and anti-miRNA). $n=3$ biologically independent experiments. For $\mathbf{b}$ and $\mathbf{d}-\mathbf{g}$, each histogram represents the mean \pm s.d., ${ }^{*} P<0.05$. For $\mathbf{e}-\mathbf{g}$, two-sided unpaired $t$-tests with Welch's correction were performed. Source data are available in Supplementary Table 8.

(Fig. 3e). The sensors contained either TYRP1-C or TYRP1-A 3' UTR fused to the luciferase-coding sequence. Luciferase activity decreased only with TYRP1-C $3^{\prime}$ UTR, confirming that miR-155 induces the decay of the $\mathrm{C}$ allele. In contrast, the $3^{\prime}$ UTR of TYRP1-A resisted endogenous miRNAs released from TYRP1 (Fig. 3e) as envisioned by the competition assay. These results highlighted the importance 
a

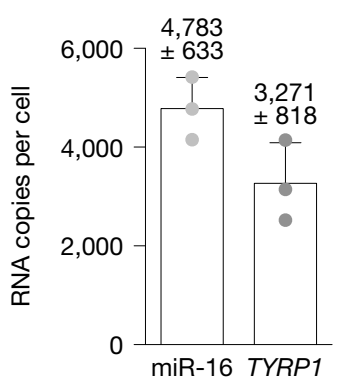

d

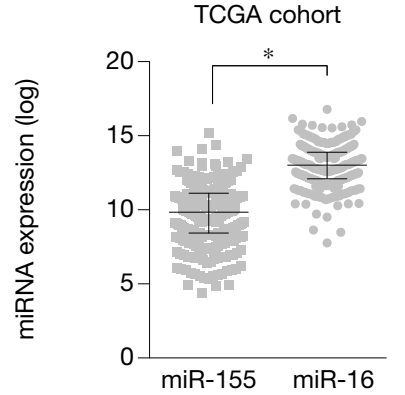

b

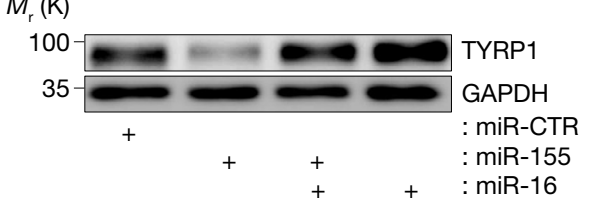

e
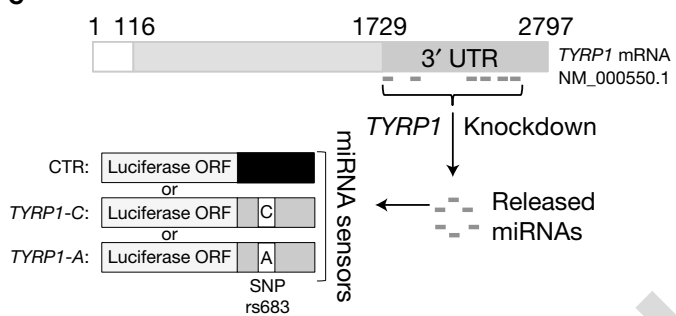

C

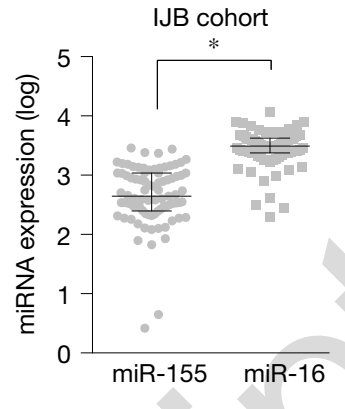

$\mathbf{f}$

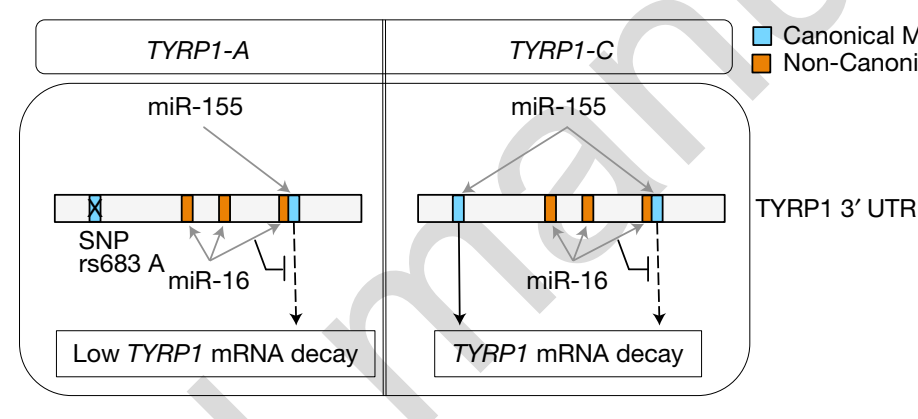

g

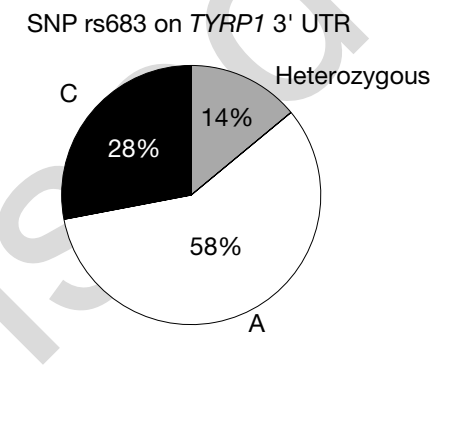

Figure 3 Biological and clinical significance of the TYRP1 mRNA as a miRNA sponge. (a) Absolute quantification of TYRP1 mRNA and miR-16 in $501 \mathrm{Mel}$ cells was determined by northern blot experiments (gels in Supplementary Fig. 2d,e). (b) Expression level of TYRP1 protein in response to synthetic miRNA alone or in combination. The image is representative of two biologically independent experiments. GAPDH serves as a loading control. Unprocessed original blots are shown in Supplementary Fig. 7. (c,d) Quantification of miR-155 and miR-16 in $n=85$ metastatic melanoma tumours (c) (IJB cohort; Supplementary Table 3 ) and in $n=349$ metastatic melanoma tumours (d) (TCGA cohort). The lines represent the median and interquartile range and two-sided paired $t$-tests were used $\left({ }^{*} P<0.0001\right)$. (e) Schematic representation of TYRP1 knockdown leading to the endogenous release of miRNA docked on TYRP1 mRNA, and the redirection of released miRNA targeting miRNA sensors (left). The miRNA sensors are an unrelated sequence (CTR); the 3' UTR of TYRP1 from NM_000550.2 (TYRP1-C); and NM_000550.1 (TYRP1-A) fused to the luciferase ORF. Luciferase activity

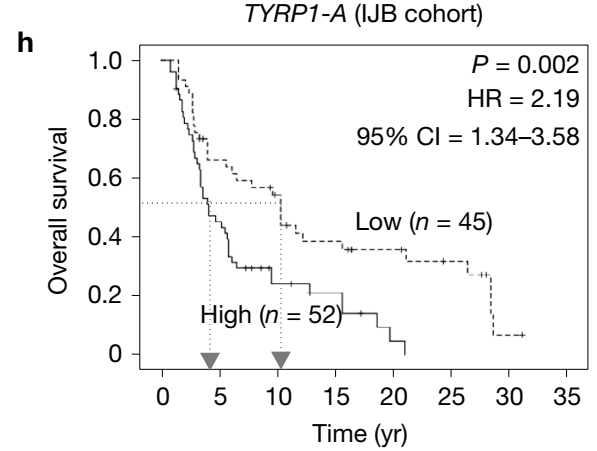

(right) was evaluated after three days of transfection. Two-sided unpaired $t$-test with Welch's correction; ${ }^{*} P<0.05$. For a and e, $n=3$ biologically independent experiments and each histogram represents the mean \pm s.d. Source data are available in Supplementary Table 8. (f) Three non-canonical MRE-16s have been identified in TYRP1-A and $-C$, and biologically validated. miR-16 binds to these sites but does not mediate TYRP1 mRNA decay. In addition, miR-16 negates miR-155-induced TYRP1 decay. The interaction of miR-155 and miR-16 with TYRP1-A leads to low TYRP1-A mRNA decay in contrast to TYRP1-C. (g) Genotyping of 174 metastatic melanoma samples from the IJB cohort (Supplementary Table 3) to determine TYRP1-A and TYRP1-C alleles (SNP rs683). (h) Determination of overall survival curves by Kaplan-Meier analysis in the TYRP1-A population of the IJB cohort, according to expression levels of TYRP1 mRNA. Arrows depict overall survival median for groups with either high ( $<5$ years) or low ( $>10$ years) TYRP1 levels. Cox regression was used to calculate $P$ values, hazard ratios (HR) and $95 \%$ confidence intervals $(\mathrm{Cl})$ for Kaplan-Meier graphs. 
of the SNP rs683 and show that the TYRP1-A allele promotes miR16 sequestration through the combination of three genetic traits: SNP rs683-A, non-canonical MRE-16s and high expression levels of TYRP1 mRNA (Fig. 3f).

To underscore the clinical relevance of TYRP1-A, we examined its prognostic value. We genotyped the 174 tumours of the Institute Jules Bordet (IJB) cohort (Supplementary Table 3) and showed, in the TYRP1-A subpopulation (58\%), a correlation between overall survival of patients and the TYRP1-A level (Fig. 3g,h).

These results emphasized the role of TYRP1-A as a miRNA sponge in metastatic melanoma through its ability to sequester the tumour suppressor miR-16.

\section{miR-16 targets}

Tumour suppressor activity of miR-16 is mediated by the mRNA decay of its RNA targets. We thus determined miR-16 targets, involved in cell proliferation and melanoma growth, when released from TYRP1 mRNA. We established the gene expression profile of melanoma cells transfected with siRNAs targeting TYRP1 (siTYRP1 no. 1-3) (Fig. 4a and Supplementary Fig. 4a). We identified 49 RNAs that were deregulated following TYRP1 KD. Gene annotation highlighted their implication in proliferation, migration and invasion processes supporting the phenotype of TYRP1 KD. We examined MAFF, NRTN, $R A B 17$ and RasGRP3, the levels of which diminished in a manner that paralleled the efficiency of TYRP1 silencing (Supplementary Fig. 4b). These four genes were also downregulated in SKMel28 TYRP1 KD cells (Supplementary Fig. 4c). To determine whether melanoma cell proliferation relied on these genes, we knocked them down using two different siRNAs and measured the cell density (Supplementary Fig. 4d). Strikingly, transient KD of NRTN, RAB17 and RasGRP3 significantly decreased the cell density, while a tendency was observed with $M A F F$ transient KD. Ectopic expression of miR-16 significantly diminished RAB17, MAFF and NRTN expression in accordance with the identification of canonical MRE-16 (Fig. 4b-d and Supplementary Fig. 4e,f). Importantly, the level of $R A B 17$ was positively correlated with that of TYRP1 in 191 metastatic melanoma samples (IJB cohort) (Fig. 4e). Comparable significant results were obtained with The Cancer Genome Atlas (TCGA) melanoma cohort for RAB17 and NRTN (Supplementary Table 4). Together, these data suggest that miR-16 is the central node of a gene network supporting its tumour-suppressor function.

\section{RAB17 promotes proliferation of melanoma cells and in vivo tumour growth}

We investigated further the biological contribution of the Ras-related RAB17 protein by silencing RAB17 expression (shRNA). RAB17 KD cells showed reduced growth ability (Fig. 4f,g). This effect was rescued following the introduction of a $R A B 17$ open reading frame (ORF), which was not targeted by RAB17 shRNA (Fig. 4h). RAB17 silencing significantly reduced the tumour growth of engrafted SKMel28 cells in immunocompromised mice, similarly to KD TYRP1 cells (Fig. 4i). These results identified RAB17 as a key downstream effector among others of the TYRP1 network involved in cell proliferation and tumour growth.

To evaluate the clinical relevance of the co-expression of TYRP1 and $R A B 17$, we analysed the overall survival of patients with metastatic melanoma according to the levels of TYRP1, RAB17 or both. We found that the $R A B 17$ mRNA level was a poor indicator of survival when analysed independently (Supplementary Fig. 5a). In contrast, its prognostic value was improved in association with TYRP1 mRNA level (Supplementary Fig. 5b). These results were confirmed in the TCGA cohort (Supplementary Fig. 5c), and the prognostic value was further improved when focusing on the TYRP1-A subpopulation (Fig. 4j). Interestingly, comparable results were obtained with the NRTN gene, adding significance to the prognostic value of TYRP1 (Supplementary Fig. 5d,e).

Together, these results support the proposed model whereby TYRP1-A mRNA regulates genes expression through miR16 sequestration.

\section{Restoring miR-16 tumour suppressor function reduces tumour growth}

Having demonstrated that TYRP1 mRNA dampens miR-16 activity, we challenged this sequestration model with small antisense oligonucleotides, known as target site blockers (TSBs) ${ }^{39-41}$. We designed two TSBs of 16 nucleotides in length: a control TSB (TSB-C1) that does not match any annotated human 3' UTR and a functional TSB (TSB-T3) that selectively binds to the sequence overlapping the TYRP1 MRE-16 site (2392-2407). The TSB-T3 sequence is fully complementary to TYRP1 mRNA and was therefore expected to bind TYRP1 with a higher affinity than miR-16, masking this MRE-16 and preventing miR-16 sequestration on TYRP1 (Fig. 5a). Accordingly, TSB-T3 increased miR-16 activity (Fig. 5b) and decreased RAB17 levels and cell density in two melanoma cell lines (Fig. 5c,d). This cell density decrease was accompanied by an increase in apoptosis (Fig. 5e). Importantly, TSB-T3 biological efficiency was correlated with TYRP1 expression levels and independent of BRAF, NRAS or TP53 mutation state (Fig. $5 \mathrm{f}$ and Supplementary Table 5 ). These data strongly indicated that TSB-T3 masks MRE-16 on TYRP1 and redirects miR-16 to RAB17 mRNA in melanoma cells expressing TYRP1.

We evaluated the therapeutic potential of TSB-T3 in a patientderived xenograft (PDX) model of melanoma (Mel006; homozygous for the TYRP1-A allele $)^{42}$. Mice were exposed to $25 \mathrm{mg} \mathrm{kg}^{-1}$ TSB-T3 or TSB-C1 daily through subcutaneous injection in the vicinity of the tumours (Fig. 6). Complete necropsy and histopathological analysis indicated that this treatment did not cause any adverse event. Consistently, mice from both groups gained weight during the course of the experiment (Fig. 6a and Supplementary Fig. 6a). Strikingly, the tumour volume was significantly lower in mice treated with TSB-T3 (Fig. 6b). The tumour volume of the control group reached $2,000 \mathrm{~mm}^{3}$ in around 13 days, while that of the TSB-T3treated group was significantly lower $\left(\sim 500 \mathrm{~mm}^{3}\right)$ and mice were still responding over 23 days (Supplementary Fig. $6 \mathrm{~b}$ ). Consistent with our hypothesis, RAB17 expression level decreased significantly in TSB-T3-treated tumours (Fig. 6c,d). To further emphasize the central role of free miR-16 in monitoring tumour growth, we examined the expression level of two well-known miR-16 targets CCND3 and YAP1 (refs 43,44). Their expressions were significantly reduced in TSBT3-treated tumours (Fig. 6e,f). Conversely, the expression of LAMP2 was not modified, as expected since $L A M P 2$ is not a miR-16 target (Supplementary Fig. 6c) (PCT/US2007/089206 and Targetscan7.1). Together, these results indicate that this therapeutic strategy is 
a

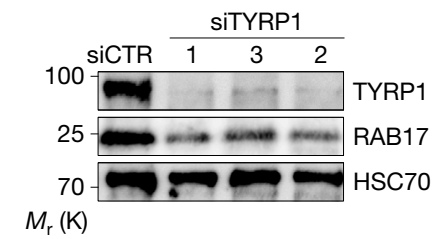

b

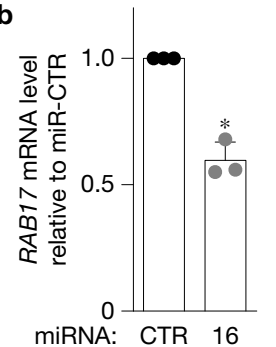

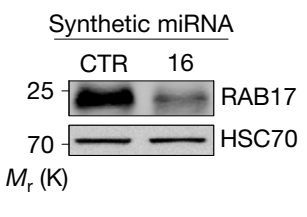

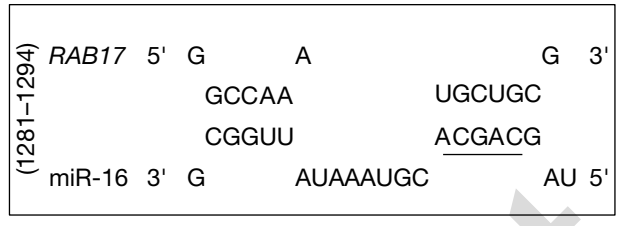

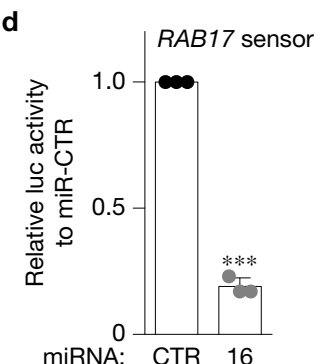

h

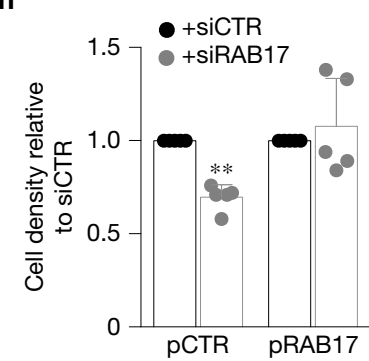

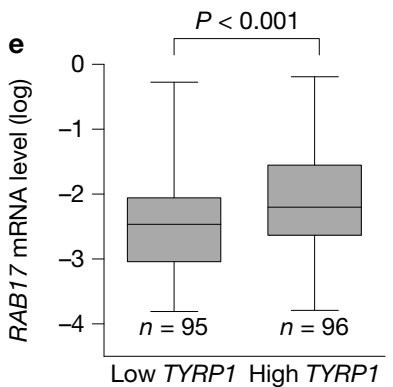

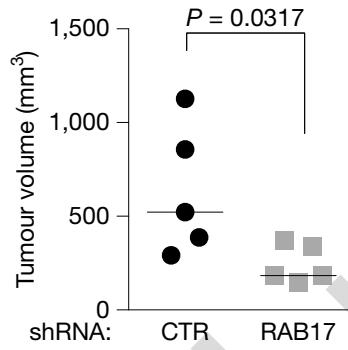

\section{f}
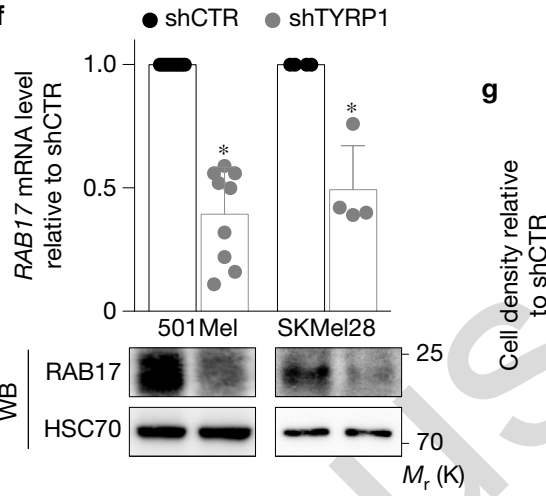

j

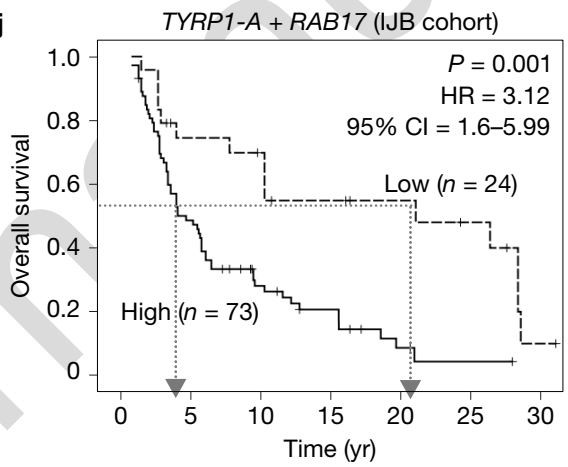

Figure 4 RAB17 promotes proliferation of melanoma cells and in vivo tumour growth. (a) TYRP1 and RAB17 protein levels in response to three different siRNAs (no. 1-3). (b) RAB17 mRNA (left; $n=3$ biologically independent experiments) and protein (right) expression in response to synthetic miR-16 or miR-CTR. (c) The MRE-16 (1281-1294) in RAB17 mRNA (NM_022449.3) was identified using RNAhybrid software ${ }^{34}$. (d) Effects of synthetic miR-16 on the RAB17 sensor (luciferase) in Huh 7.5 Drosha knockout cells ( $n=3$ biologically independent experiments). (e) Positive correlation between RAB17 and TYRP1 mRNA in tumours (IJB cohort; Supplementary Table 3). The box plot shows RAB17 expression levels measured in cells with either low or high TYRPI expression according to the TYRP1 median. The boxes represent the median, and the first and third quartiles, and the whiskers represent the minimum and maximum of all data points. Two-sided Mann-Whitney test; Pearson correlation $(r)$ is 0.5776 with $P<0.0001$. (f) $R A B 17$ mRNA (top) and protein (bottom) expression levels in response to RAB17 KD in $501 \mathrm{Mel}$ and SKMel28 cells ( $n=9$ and 4 biologically independent experiments, respectively). (g) Cell

efficient to reset miR-16 activity in a clinically relevant in vivo model (Fig. 6g).

\section{DISCUSSION}

We demonstrated herein that TYRP1 mRNA exhibits a non-coding function in melanoma by sequestering miR-16 and dampening its tumour-suppressor activity. Sequestered miR-16 is no longer able to repress its targets implicated in cell proliferation and tumour growth. density in response to $R A B 17 \mathrm{KD}$ ( $n=4$ and 3 biologically independent experiments, respectively). (h) Rescue experiments by overexpressing $R A B 17$ ORF (pRAB17) or an empty vector (pCTR) in $501 \mathrm{Mel}$ cells in the presence of either siRAB17 or siCTR ( $n=5$ biologically independent experiments). (i) Tumour volume at day 10 of shCTR or shRAB17 SKMel28 cells in nude mice ( $n=5$ mice per group). The bars correspond to the median; two-sided Mann-Whitney test; significant for $P<0.05$. (j) Determination of overall survival curves by Kaplan-Meier analysis in the TYRP1-A population (Supplementary Table 3), according to the expression level of TYRP1 and RAB17 mRNAs (Supplementary Fig. 5b for groups' definition). Cox regression was used to calculate $P$ values, hazard ratios (HR) and $95 \%$ $\mathrm{Cl}$. For $\mathbf{b}, \mathbf{d}$ and $\mathbf{f}-\mathbf{h}$, each histogram represents the mean \pm s.d.; twosided unpaired $t$-tests with Welch's correction $\left(^{*} P<0.05\right.$; ${ }^{* *} P<0.01$; ${ }^{* * *} P<0.001$ ). Western blot results are representative of at least three experiments and HSC70 serves as a loading control. Source data are available in Supplementary Table 8 and unprocessed original blots are shown in Supplementary Fig. 7.

We therefore conclude that TYRP1, through its ability to sequester miR-16, indirectly regulates melanoma growth. These results support the previously established link between TYRP1 mRNA levels and the survival of patients with metastatic melanoma ${ }^{28,29}$.

Our data identify TYRP1 mRNA as a robust miR-16 sponge. Three properties support this function. First, TYRP1 is highly expressed in most melanoma ${ }^{28,29,45,46}$. Second, the SNP rs683 genetically determines TYRP1 miRNA sponge activity. The SNP rs683-A negates 
a

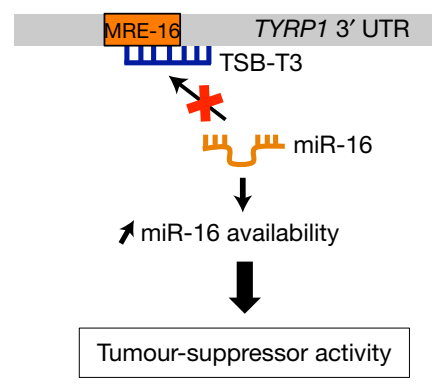

b

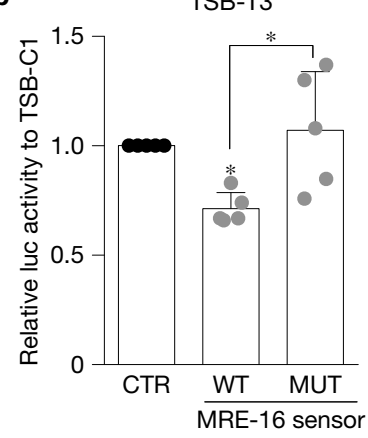

c

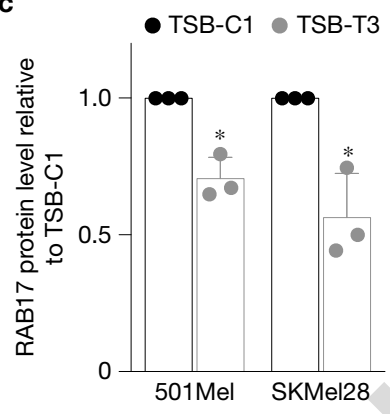

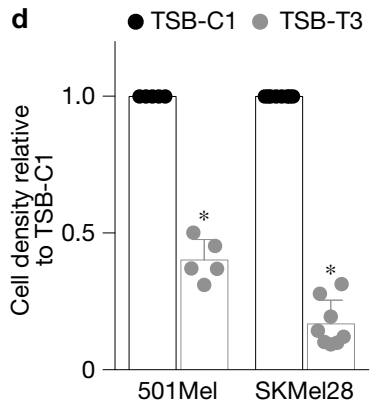

f

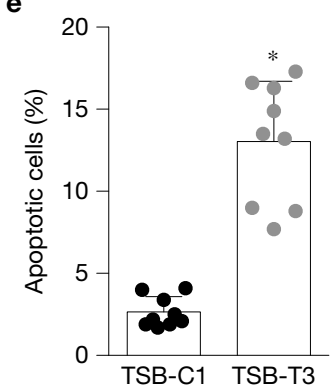

Figure 5 Target site blocker restores miR-16 function. (a) Competition model between target site blocker (TSB) and miR-16: TSB-T3 avoids miR-16 sequestration on TYRP1 mRNA. TSB-T3 selectively binds to the sequence overlapping the miR-16 site (NM_000550.1, nucleotides 2392-2407) in the $3^{\prime}$ UTR of TYRP1. TSB-C1 presents no significant match to any annotated human $3^{\prime}$ UTR. (b) Effects of TSB-T3 (via free endogenous miR-16) on wild-type (WT) or mutated (MUT) MRE-16 sensors in $501 \mathrm{Mel}$ cells. Luciferase activity was evaluated after two days of transfection ( $n=5$ biologically independent experiments; oneway ANOVA with Holm-Sidak's multiple comparisons test, ${ }^{*} P<0.05$ ). (c) Western-blot quantification of RAB17 protein levels in $501 \mathrm{Mel}$ and SKMel28 cells transfected with TSB-C1 or TSB-T3 $(n=3$ biologically independent experiments; ${ }^{*} P<0.05$ ). (d) Cell density of $501 \mathrm{Mel}$ and

miR-155-dependent mRNA decay of TYRP1-A, limiting TYRP1-A mRNA turnover ${ }^{27,30}$. Third, non-canonical miR-16-binding sites sequester miR-16. These non-canonical MRE-16s have motifs that differ from the top-ranking GCUGCU motif, which is known to interact with the miR-16 seed sequence ${ }^{4,7,35}$. They also differ from the recently published non-canonical site types (NSTs), which, in a few cases, can promote a moderate mRNA decay ${ }^{5}$. We indeed demonstrated that these non-canonical MREs prime miR-16 binding on TYRP 1 mRNA and promote miRNA sequestration since miR-16 does not cause TYRP1 mRNA decay and induces an unusual increase of TYRP1 mRNA. Competition experiments suggest that miR-16 binding alters miR-155-induced TYRP1 decay. Although the underpinning mechanism remains unknown, we speculate that the vicinity of MRE-16 no. 3 and MRE-155 no. 3 that is conserved in mouse and human may be partly responsible. This hypothesis is supported by recent findings showing that the overall proficiency of an MRE is determined by the context features of an miRNA and its mRNA target ${ }^{4,5,47-49}$. Together, our results provide molecular evidence that non-canonical MREs are central regulators of miRNA activity.

This study strengthened the finding that the cellular abundance of miRNAs can be a poor indicator of their RNA-decay activity

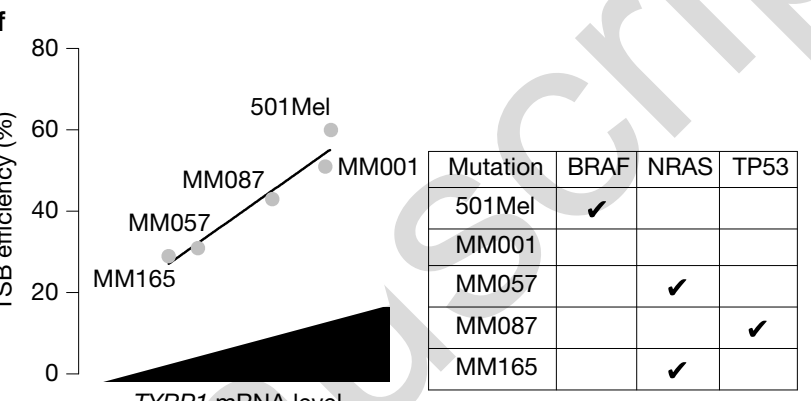

TYRP1 mRNA level
SKMel28 ( $n=5$ and 8 biologically independent experiments, respectively) in response to target site blocker (TSB-C1 or TSB-T3) transfection at day 2; $* P<0.001$. (e) Apoptotic cells (\%) in response to target site blocker (TSB-C1 or TSB-T3) $24 \mathrm{~h}$ after transfection in $501 \mathrm{Mel}$ cells $(n=3$ biologically independent experiments; ${ }^{*} P<0.001$ ). (f) Positive correlation between TSB-T3 efficiency on cell density (\%) and TYRP1 mRNA level (reported in Supplementary Table 5). The table (right) summarizes the genomic alterations (mutations) on BRAF, NRAS and TP53 described in these samples ${ }^{42} . n=5$ cell lines or short-term cultures; Spearman correlation $r=0.9747$ and $P=0.0333$; significant for $P<0.05$. For $\mathbf{b}-\mathbf{e}$, each histogram represents the mean $\pm \mathrm{s}$.d. For $\mathbf{c}-\mathbf{e}$, two-sided unpaired $t$-tests with Welch's correction were performed. Source data are available in Supplementary Table 8.

as already envisioned ${ }^{4,5,24,50,51}$. miRNA sequestration significantly reduces the active and free fraction of a given miRNA. In our study, the levels of miR-16 are comparable between tumours and do not predict the clinical outcome of patients with metastatic melanoma (Supplementary Fig. 5f,g). This contrasts to the levels of the miR-16 sponge (TYRP1 mRNA), either itself or in association with one of the miR-16 targets (RAB17 mRNA or NRTN mRNA), adding significance to the sponge prognostic value. These data warrant further investigation to better determine the fraction of active miRNAs per cell and demonstrate that more accurate prediction of patient survival can be obtained by quantifying three parameters dictating the sponge mechanism instead of the miR-16 levels.

Importantly, we developed three proof-of-concept approaches to restore the tumour-suppressor activity of miR-16 in melanoma. Sequestration of miR-16 can be abolished by RNA interference eradicating the miR-16 sponge or by saturating the TYRP1-sequestration capacity with synthetic miR-16. The most elegant approach is certainly through the use of TSBs reducing tumour growth in a preclinical in vivo model. Further investigation is now required to determine whether combining several TSBs targeting distinct non-canonical MRE-16 on TYRP1 will potentiate the biological effect. 

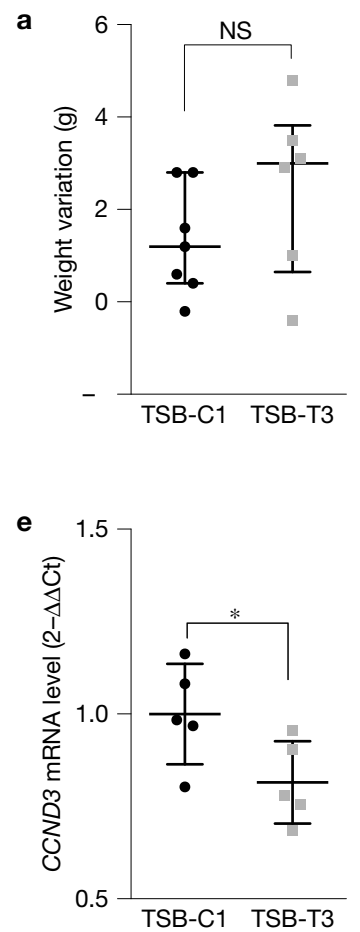

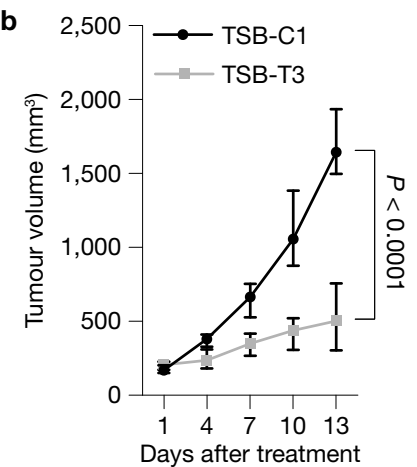

$c$

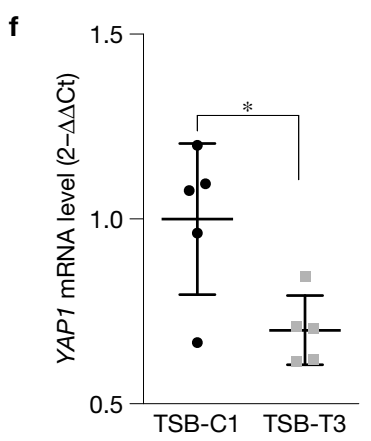

g
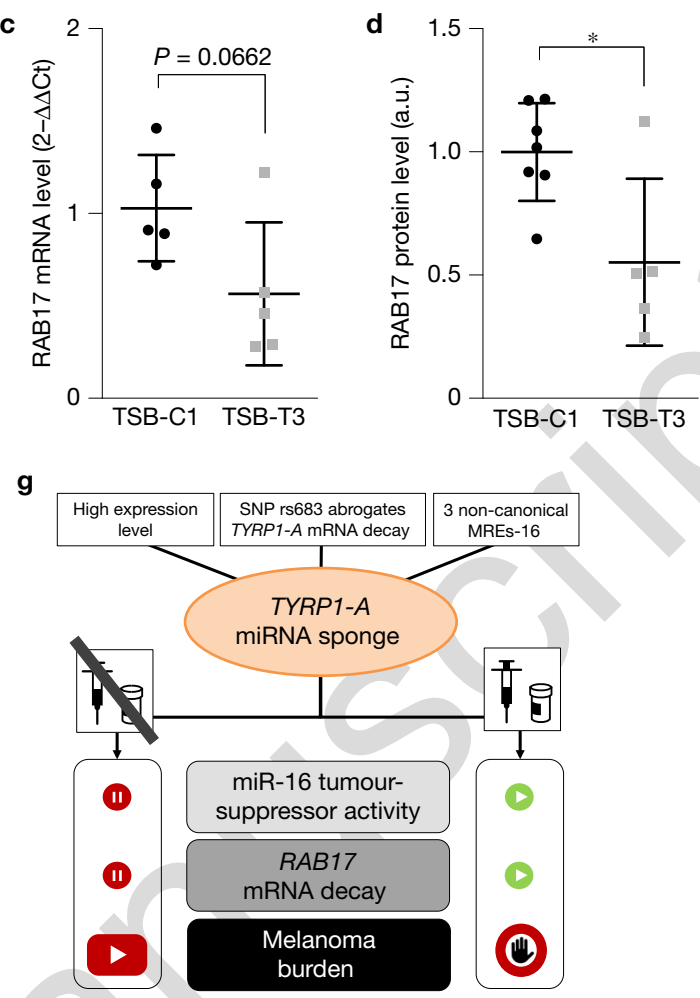

Figure 6 Restoring miR-16 tumour-suppressor function reduces tumour growth. (a) Weight variation of patient-derived tumour xenograft (PDX) mice treated with TSB-C1 or TSB-T3 ( $n=7$ or 6 mice, respectively; twosided Mann-Whitney test; NS, not significant). (b) Tumour volume of PDX mice treated with TSB-C1 or TSB-T3 ( $n=7$ or 6 mice, respectively; twoway ANOVA without adjustment). (c) Quantification of RAB17 mRNA in melanoma tumours treated with TSB-C1 or TSB-T3 ( $n=5$ mice per group; $P=0.0662$ ). (d) Quantification of RAB17 protein levels in tumours treated as described in b. a.u., arbitrary unit by western blot analyses $(n=7$ or 5 mice, respectively). For $\mathbf{a}, \mathbf{b}$, the graphs plot the median and the interquartile range. (e,f) Quantification of $C C N D 3$ (e) or YAP1 (f) mRNA in melanoma tumours treated with TSB-C1 or TSB-T3 $(n=5$ mice per group). For $\mathbf{c}-\mathbf{f}$, the lines represent the mean \pm s.d.; values represent fold change relative to the mean of the TSB-C1 condition; two-sided unpaired $t$-tests with Welch's correction; ${ }^{*} P<0.05$. Source data are available in
Supplementary Table 8. (g) Model for the sponge activity of TYRP1-A mRNA in melanoma and therapeutic strategy. The miRNA sponge activity is tightly associated with the clinical outcome of patients with metastatic melanoma, and is dependent on TYRP1-A expression levels. High levels of TYRP1-A mRNA are dictated by the SNP rs683 and non-canonical MREs. SNP rs683-A strongly decreases the miR-155-dependent mRNA decay of TYRP1-A. TYRP1-A mRNA can sequester miR-16 on non-canonical MRE-16, limiting its tumour-suppressor activity by cancelling its usual mRNA decay activity (that is, mRNA decay of RAB17). This harmful mechanism may be unlocked by either destroying the miR-16 sponge by RNAi; increasing the total amount of miR-16 per cell, thus bypassing the sequestration capacity of TYRP1-A; or by avoiding the miR-16 sequestration on TYRP1-A by using small oligonucleotide competitors (target site blockers). We therefore propose three original ways to overcome melanoma addiction to the miR-16 sponge.
Restoration of miR-16 RNA-decay activity leads to a potent 'antimelanoma effect', which is herein the consequence of a sum of RNA decay and protein downregulation. In other words, the tumour suppressor miR-16 is an upstream regulator of several downstream targets such as RAB17 and NRTN that regulate cell proliferation. The role of RAB17 in tumour growth warrants further investigations in light of the ones recently identified for RAB7a and RAB5c in melanoma ${ }^{52,53}$. Finally, the importance of such a cascade mechanism relies on the expression level of the sponge, the amount of the sequestered miRNA and the biological function of secondary targets ${ }^{24}$.

We demonstrate here that TSBs can be used efficiently in vivo to terminate sponge-mediated sequestration of miRNAs. Given the recent renewed interest in antisense drugs, such as KYNAMRO (mipomersen), SPINRAZA (nusinersen) or ASO-targeting long noncoding RNAs such as $S A M M S O N^{42,54,55}$, our findings open further therapeutic opportunities through the use of TSBs as innovative therapeutic molecules.

\section{METHODS}

Methods, including statements of data availability and any associated accession codes and references, are available in the online version of this paper.

Note: Supplementary Information is available in the online version of the paper

\section{ACKNOWLEDGEMENTS}

The authors thank the Gene Expression and Oncogenesis team, especially K. Tutoré, for helpful discussions; N. Cougot, R. Gillet, A. Méreau and E. Giudice from the CNRS UMR6290; the Rennes FHU CAMIn team; and B. Turlin, J. Le Seyec, M. Trotard and A. Popa for providing scientific expertise. The authors acknowledge the SFR Biosit core facilities of Rennes University with the histopathology $\mathrm{H}^{2} \mathrm{P}^{2}$ (A. Fautrel) and cell imaging ImPACcell (R. Le Guevel) platforms, and the ARCHE animal housing facility for their help and support. The authors also thank the Genomic platform from the translational research department of the Curie Institute in Paris. This study received financial support from the following: Institut National du Cancer PAIR Melanoma program; Ouest Valorisation; Ligue National Contre le Cancer (LNCC) Départements du Grand-Ouest; Région Bretagne; University of Rennes 1; CNRS; Association Transfusion Sanguine et Biogénétique Gaétan Saleun; MEDIC Foundation; Les Amis de l'Institut Bordet; Fondation Lambeau-Marteaux; 
European Organisation for Research and Treatment of Cancer Tournesol program; and the Société Française de Dermatologie. Further support was provided by a 'Ligue Nationale Contre le Cancer' (LNCC) fellowship (E.D.-F.) and from the Région Bretagne and the LNCC Grand Ouest (A.G.; M.M.) and from Faculté des Sciences Pharmaceutiques de l'Université de Rennes 1 (M.M.). The authors are grateful to G. Lizée for providing the Mel624 cell line, to R. B. Darnell for providing Huh7.5 Drosha KO cells, to J. Wrana for pMS2 vectors and to V. J. Hearing for the antiTYRP1 (PEP1) antibody.

\section{AUTHOR CONTRIBUTIONS}

Conceptualization: D.G., M.M. and M.-D.G.; methodology: D.G., M.M., L.B., A.R., A.M., N.M., M.-L.P.-M., T.M., S.C., B.F. and S.T.-D.; investigation: D.G., M.M., L.B., F.J., A.R., E.D.-F., A.M., N.M., M.-L.P.-M., T.M., S.C., A.G. and R.B.J.; formal analysis: D.G., M.M., F.J., A.R., N.M., B.M. and F.R.; writing-original draft: D.G., M.M. and M.-D.G.; funding acquisition: D.G., F.J., T.M., S.C., G.G. and M.-D.G.; resources: F.J., F.R., P.E., J.-C.M. and G.G.; supervision: D.G. and M.-D.G.

\section{COMPETING FINANCIAL INTERESTS}

The authors declare no competing financial interests.

Published online at http://dx.doi.org/10.1038/ncb3623

Reprints and permissions information is available online at www.nature.com/reprints Publisher's note: Springer Nature remains neutral with regard to jurisdictional claims in published maps and institutional affiliations.

1. Hausser, J. \& Zavolan, M. Identification and consequences of miRNA-target interactions-beyond repression of gene expression. Nat. Rev. Genet. 15, 599-612 (2014).

2. Bartel, D. P. MicroRNAs: target recognition and regulatory functions. Cell 136, 215-233 (2009)

3. Chi, S. W., Hannon, G. J. \& Darnell, R. B. An alternative mode of microRNA target recognition. Nat. Struct. Mol. Biol. 19, 321-327 (2012).

4. Agarwal, V., Bell, G. W., Nam, J.-W. \& Bartel, D. P. Predicting effective microRNA target sites in mammalian mRNAs. eLife 4, e05005 (2015).

5. Kim, D. et al. General rules for functional microRNA targeting. Nat. Genet. 48, 1517-1526 (2016).

6. Loeb, G. B. et al. Transcriptome-wide miR-155 binding map reveals widespread noncanonical microRNA targeting. Mol. Cell 48, 760-770 (2012).

7. Helwak, A., Kudla, G., Dudnakova, T. \& Tollervey, D. Mapping the human miRNA interactome by CLASH reveals frequent noncanonical binding. Cell 153, 654-665 (2013).

8. Khorshid, M., Hausser, J., Zavolan, M. \& van Nimwegen, E. A biophysical miRNAmRNA interaction model infers canonical and noncanonical targets. Nat. Methods 10, 253-255 (2013).

9. Grosswendt, S. et al. Unambiguous identification of miRNA: target site interactions by different types of ligation reactions. Mol. Cell 54, 1042-1054 (2014).

10. Ebert, M. S., Neilson, J. R. \& Sharp, P. A. MicroRNA sponges: competitive inhibitors of small RNAs in mammalian cells. Nat. Methods 4, 721-726 (2007).

11. Franco-Zorrilla, J. M. et al. Target mimicry provides a new mechanism for regulation of microRNA activity. Nat. Genet. 39, 1033-1037 (2007).

12. Giusti, S. A. et al. MicroRNA-9 controls dendritic development by targeting REST. eLife 3, 1-22 (2014).

13. Reichel, M., Li, Y., Li, J. \& Millar, A. A. Inhibiting plant microRNA activity: molecular SPONGEs, target MIMICs and STTMs all display variable efficacies against target microRNAs. Plant Biotechnol. J. 13, 915-926 (2015).

14. Tay, Y. et al. Coding-independent regulation of the tumor suppressor PTEN by competing endogenous mRNAs. Cell 147, 344-357 (2011).

15. Poliseno, L. et al. A coding-independent function of gene and pseudogene mRNAs regulates tumour biology. Nature 465, 1033-1038 (2010).

16. Karreth, F. A. et al. In vivo identification of tumor-suppressive PTEN ceRNAs in an oncogenic BRAF-induced mouse model of melanoma. Cell 147, 382-395 (2011).

17. Karreth, F. A. et al. The BRAF pseudogene functions as a competitive endogenous RNA and induces lymphoma in vivo. Cell 161, 319-332 (2015).

18. Thomson, D. W. \& Dinger, M. E. Endogenous microRNA sponges: evidence and controversy. Nat. Rev. Genet. 17, 272-283 (2016).

19. Tay, Y., Rinn, J. \& Pandolfi, P. P. The multilayered complexity of ceRNA crosstalk and competition. Nature 505, 344-352 (2014).

20. Guo, J. U., Agarwal, V., Guo, H. \& Bartel, D. P. Expanded identification and characterization of mammalian circular RNAs. Genome Biol. 15, 409 (2014).

21. Memczak, S., Jens, M., Elefsinioti, A. \& Torti, F. Circular RNAs are a large class of animal RNAs with regulatory potency. Nature 495, 333-338 (2013).

22. Hansen, T. B. et al. Natural RNA circles function as efficient microRNA sponges. Nature 495, 384-388 (2013).

23. Zheng, Q. et al. Circular RNA profiling reveals an abundant circHIPK3 that regulates cell growth by sponging multiple miRNAs. Nat. Commun. 7, 11215 (2016).
24. Migault, M., Donnou-Fournet, E., Galibert, M.-D. \& Gilot, D. Definition and identification of small RNA sponges: focus on miRNA sequestration. Methods 117 , 35-47 (2017).

25. Boissy, R. E., Sakai, C., Zhao, H., Kobayashi, T. \& Hearing, V. J. Human tyrosinase related protein-1 (TRP-1) does not function as a DHICA oxidase activity in contrast to murine TRP-1. Exp. Dermatol. 7, 198-204 (1998).

26. Ghanem, G. \& Journe, F. Tyrosinase related protein 1 (TYRP1/gp75) in human cutaneous melanoma. Mol. Oncol. 5, 150-155 (2011).

27. El Hajj, P. et al. SNPs at miR-155 binding sites of TYRP1 explain discrepancy between mRNA and protein and refine TYRP1 prognostic value in melanoma. Br. J. Cancer 113, 91-98 (2015).

28. El Hajj, P. et al. Tyrosinase-related protein 1 mRNA expression in lymph node metastases predicts overall survival in high-risk melanoma patients. $\mathrm{Br}$. J. Cancer 108, 1641-1647 (2013).

29. Journe, F. et al. TYRP1 mRNA expression in melanoma metastases correlates with clinical outcome. Br. J. Cancer 105, 1726-1732 (2011)

30. $\mathrm{Li}, \mathrm{J}$. et al. Evidence for positive selection on a number of microRNA regulatory interactions during recent human evolution. PLoS Genet. 8, 1-12 (2012).

31. Kittler, R. et al. Genome-scale RNAi profiling of cell division in human tissue culture cells. Nat. Cell Biol. 9, 1401-1412 (2007).

32. Li, C.-Y. Y. et al. The effect of antisense tyrosinase-related protein 1 on melanocytes and malignant melanoma cells. Br. J. Dermatol. 150, 1081-1090 (2004).

33. Tominaga, $\mathrm{K}$. et al. Competitive regulation of nucleolin expression by HuR and miR-494 . Mol. Cell. Biol. 31, 4219-4231 (2011).

34. Rehmsmeier, M., Steffen, P., Hochsmann, M. \& Giegerich, R. Fast and effective prediction of microRNA/target duplexes. RNA 10, 1507-1517 (2004).

35. Selbach, M. et al. Widespread changes in protein synthesis induced by microRNAs Nature 455, 58-63 (2008)

36. Pekarsky, Y. \& Croce, C. M. Role of miR-15/16 in CLL. Cell Death Differ. 22 6-11 (2014).

37. Poell, J. B. et al. A functional screen identifies specific microRNAs capable of inhibiting human melanoma cell viability. PLOS ONE 7, e43569 (2012).

38. Ebert, M. S. \& Sharp, P. A. MicroRNA sponges: progress and possibilities. RNA 16 2043-2050 (2010)

39. Hartmann, P. et al. Endothelial Dicer promotes atherosclerosis and vascular inflammation by miRNA-103-mediated suppression of KLF4. Nat. Commun. 7, 10521 (2016).

40. Wynendaele, J. et al. An illegitimate microRNA target site within the 3' UTR of MDM4 affects ovarian cancer progression and chemosensitivity. Cancer Res. 70, 9641-9649 (2010).

41. Messina, A. et al. A microRNA switch regulates the rise in hypothalamic GnRH production before puberty. Nat. Neurosci. 19, 835-844 (2016).

42. Leucci, E. et al. Melanoma addiction to the long non-coding RNA SAMMSON. Nature $531,518-522$ (2016)

43. Liu, Q. et al. miR-16 family induces cell cycle arrest by regulating multiple cell cycle genes. Nucleic Acids Res. 36, 5391-5404 (2008).

44. Kang, W. et al. Targeting of YAP1 by microRNA-15a and microRNA-16-1 exerts tumor suppressor function in gastric adenocarcinoma. Mol. Cancer 14, 1-10 (2015).

45. Falkenius, J. et al. High expression of glycolytic and pigment proteins is associated with worse clinical outcome in stage III melanoma. Melanoma Res. 23, 452-460 (2013).

46. Rose, A. A. N. et al. MAPK pathway inhibitors sensitize BRAF-mutant melanoma to an antibody-drug conjugate targeting GPNMB. Clin. Cancer Res. 22, 6088-6098 (2016).

47. Grimson, A. et al. MicroRNA targeting specificity in mammals: determinants beyond seed pairing. Mol. Cell 27, 91-105 (2007).

48. Garcia, D. M. et al. Weak seed-pairing stability and high target-site abundance decrease the proficiency of Isy- 6 and other microRNAs. Nat. Struct. Mol. Biol. 18, 1139-1146 (2011).

49. Arvey, A., Larsson, E., Sander, C., Leslie, C. S. \& Marks, D. S. Target mRNA abundance dilutes microRNA and siRNA activity. Mol. Syst. Biol. 6, 363 (2010).

50. Luna, J. M. et al. Hepatitis C virus RNA functionally sequesters miR-122. Cell 160, 1099-1110 (2015).

51. Leung, A. K. L. The whereabouts of microRNA actions: cytoplasm and beyond. Trends Cell Biol. 25, 601-610 (2015).

52. Alonso-Curbelo, D. et al. RAB7 controls melanoma progression by exploiting a lineage-specific wiring of the endolysosomal pathway. Cancer Cell 26, 61-76 (2014)

53. Vizoso, M. et al. Epigenetic activation of a cryptic TBC1D16 transcript enhances melanoma progression by targeting EGFR. Nat. Med. 21, 741-750 (2015).

54. Raal, F. J. et al. Mipomersen, an apolipoprotein B synthesis inhibitor, for lowering of LDL cholesterol concentrations in patients with homozygous familial hypercholesterolaemia: a randomised, double-blind, placebo-controlled trial. Lancet 375, 998-1006 (2010).

55. Finkel, R. S. et al. Treatment of infantile-onset spinal muscular atrophy with nusinersen: a phase 2, open-label, dose-escalation study. Lancet $\mathbf{3 8 8}$, 3017-3026 (2016). 


\section{METHODS}

Cell lines and culture conditions. Metastatic melanoma (MM) cell lines were derived from tumours by the Laboratory of Oncology and Experimental Surgery (G.G.) at the Institute Jules Bordet, Brussels ${ }^{27-29,56}$ (Supplementary Tables 1 and 5). MM lines were propagated in humidified air $\left(37^{\circ} \mathrm{C}, 5 \% \mathrm{CO}_{2}\right)$ in flasks containing HAM-F10 medium supplemented with $5 \%$ heat-inactivated fetal bovine serum (FBS), 5\% heat-inactivated newborn calf serum, L-glutamine and $1 \%$ penicillinstreptomycin antibiotics (all from Gibco, ThermoFisher Scientific). 501Mel, ME1402 and SKMel28 cell lines were obtained from ATCC and routinely tested for mycoplasma contamination. SKMel28-luc were obtained from T.M. (INSERM U1078, France) ${ }^{57}$ and Mel624 were kindly provided by G. Lizée (the University of Texas, MD Anderson Cancer Center). Mouse melanoma B16-F10-luc-G5 cells were obtained from PerkinElmer. They were grown in humidified air $\left(37^{\circ} \mathrm{C}, 5 \% \mathrm{CO}_{2}\right)$ and in RPMI 1640 medium (Gibco) supplemented with 10\% FBS (PAA) and 1\% penicillin-streptomycin. Huh7.5 Drosha knockout cells were kindly provided by R. B. Darnell (Rockefeller University, New York, USA), and grown as previously described $^{50}$.

Xenograft. Mice were maintained under specific pathogen-free conditions in our accredited animal house (A 35-238-40). The animal study follows the 3R (replacereduce-refine) framework and has been filed with and approved by the French Government Board (No. 04386.03). Animal welfare is a constant priority: animals were thus euthanized under anaesthesia. A total of 1-3.10 $0^{6}$ SKMel28-luc or SKMel28 cells were injected subcutaneously into female NMRI nude mice and tumour growth was assessed as previously described ${ }^{57}$. This study is compliant with all relevant ethical regulations regarding animal research.

Pharmacological treatment of mice. The Mel006 PDX model derived from a human metastatic melanoma lesion carrying the BRAF(V600E) mutation and was treatment-naive. Written informed consent was obtained from patients and all procedures involving human samples were approved by the UZ Leuven/KU Leuven Medical Ethical Committee (ML8713/S54185). All procedures involving animals (NMRI nude, $\sim 6$-week-old females) were performed in accordance with the guidelines of the Catholic University of Leuven (KU Leuven) Animal Care and Use Ethical Committee (P147/2012 and P038/2015). Once tumours reached $180 \mathrm{~mm}^{3}$ (median, 139-240), $25 \mathrm{mg} \mathrm{kg}^{-1}$ of TSB-T3 or control TSB-C1 was subcutaneously injected at the vicinity of the tumours every day. No specific randomization method was used and no statistical method was used to predetermine sample size. According to animal welfare guidelines, mice have to be killed when tumours reach a volume of $2,000 \mathrm{~mm}^{3}$ or when their body weight decreases more than $20 \%$ from the initial weight. Mice used in this paper never reached or overcame these limits. The investigators were blinded for the evaluation of the results. This study is compliant with all relevant ethical regulations regarding animal research and research involving human participants.

Tissues. Cutaneous and lymph node metastases $(n=191)$ were collected from patients with stages III and IV melanoma undergoing surgery as a part of the diagnostic work-up or therapeutic strategy at the Institute Jules Bordet (Brussels) between 1998 and 2009. Samples were collected randomly, with no inclusion or exclusion criteria. Immediately after surgery, specimens were snap-frozen in liquid nitrogen and stored at $-80^{\circ} \mathrm{C}$ until use. This study was compliant with all relevant ethical regulations regarding research involving human participants and approved by the IJB ethics committee. Informed consent was obtained from all subjects. The majority of the melanomas were of superficial spreading or nodular histological subtypes with Breslow's thicknesses $>1 \mathrm{~mm}$. The clinical characteristics of the patients and tissues are outlined in Supplementary Table 3.

Target site blockers. miRCURY LNA TSBs were designed by Exiqon ${ }^{39-41}$. TSB-T3 selectively binds to a sequence overlapping the MRE-16 no. II site (Supplementary Table 6). TSB-C1, the control TSB, has no significant match to any annotated human $3^{\prime}$ UTR.

Plasmids, siRNA and miRNA. Sequences are available in Supplementary Table 6. Luciferase reporter plasmids (Ambion pMIR-REPORT luciferase containing control sequences or $3^{\prime}$ UTR TYRP1-A or TYRP1-C) were kindly provided by J. Wrana, University of Toronto, Canada ${ }^{30}$. Individual MRE-16s, individual MRE-155s and MRE-16 sensor sequences ${ }^{58}$ were cloned into the pLightSwitch plasmid (SwitchGear Genomics, Ozyme) using gBlocks (IDT) (Supplementary Table 6). The RAB17 ORF was cloned into pGreenPuro vector (Ozyme) and the synthetic sequence was designed to resist siRAB17 (TCGGTACGTGAAGAACGAC $=>$ AAGATATGTCAAAAATGAT). Full-length TYRP1 was also cloned into pGreenPuro vector, and the synthetic sequence was designed to resist shTYRP1 (CCTGGGATACACTTATGAA $=>$ CCTCGGTTATACGTACGAG). The MS2-eGFP and control pMS2-lacZ vectors used for MS2-RIP were obtained from
N. Cougot (IGDR-CNRS UMR 6290, Rennes, France). pMS2-3' UTR TYRP1-A was obtained by cloning PCR products from the plasmid kindly provided by J. Wrana (Supplementary Table 6). Vectors were verified by sequencing. Synthetic miRNAs, miRNA inhibitors and siRNAs used are described in Supplementary Table 6. Transfections were performed using Lipofectamine 2000 reagent or Lipofectamine RNAiMAX (ThermoFisher Scientific) according to the manufacturer's instructions.

shRNA experiments. Lentiviral particles carrying shRNA vectors targeting human TYRP1 mRNA (shTYRP1, TRCN0000118673), RAB17 mRNA (shRAB17, TRCN0000048017) and scrambled shRNA (shCTR, SHC002V) were purchased from Sigma-Aldrich. Lentiviral production was performed as recommended (http://tronolab.epfl.ch) using 293T cells. After infection, cells were maintained in the presence of puromycin for selection (Invivogen).

RNA extraction and reverse transcription. RNA was extracted from cell samples using a NucleoSpin RNAII kit (Macherey-Nagel) and quantified using a NanoDrop 1000 Spectrophotometer (ThermoFisher Scientific). Reverse transcription was performed using a High-Capacity cDNA Reverse Transcription kit (Applied Biosystems). For microRNA quantification, total RNA was extracted using mirVana miRNA Isolation Kit (Life Technologies). Reverse transcription was performed using a TaqMan microRNA RT kit and megaplex RT primers (Life Technologies).

Absolute quantification of RNA by northern blot. TYRP1 mRNA used for the standard range was transcribed from TYRP1-PCR-amplified templates (generated using a plasmid containing TYRP1 $3^{\prime}$ UTR and a forward primer containing T7 promoter sequence) with the MEGAscript T7 kit (Ambion). RNA was gel-purified, eluted and ethanol-precipitated. Single-strand miR-16-5p used for the standard range was synthesized by IDTDNA. Ten to thirty micrograms of denatured total RNA was separated on either $1 \%$ agarose gel with $6.5 \%$ formaldehyde for mRNA detection, or on $15 \%$ PAGE gel containing $8 \mathrm{M}$ urea for miRNA detection, and transferred onto NYTRAN or Hybond-N+ membranes (Amersham). After UV crosslinking, membranes were pre-hybridized with ExpressHyb (ClonTech) and hybridized with a TYRP1 probe labelled by random priming with $\left[\alpha-{ }^{32} \mathrm{P}\right] \mathrm{dCTP}$ (Rediprime II DNA labelling system; Amersham) or with GAPDH miR-16, and U6 probes labelled by T4 Polynucleotide Kinase (New England Biolabs) with $\left[\gamma^{32}{ }^{32}\right.$ P]ATP. TYRP1 probe was synthesized by IDT (Supplementary Table 6). Signals were revealed with a Typhoon FLA 9500 scanner (GE Healthcare). Quantifications were performed using ImageJ software (https://imagej.nih.gov/ij).

Relative quantitative PCR. Quantitative PCR was performed on $1 \mathrm{ng}$ cDNA, in 384-well plates using the SYBR Green PCR Master Mix (Applied Biosystems) with the 7900HT Fast Real-Time PCR System (Applied Biosystems). Relative amounts of transcripts were determined using the $\Delta \Delta-\mathrm{Ct}$ method and human $18 \mathrm{~S}$ or GAPDH transcript level was used as an internal control for each cell line sample. $S 100 b$ was used as an internal control for samples from tumours ${ }^{29}$ (Supplementary Table 6). TaqMan assays were performed as recommended by the manufacturer (Applied Biosystems). Relative amounts of transcripts were determined using the $\Delta-\mathrm{Ct}$ method and human RNU6B was used as an internal control for each cell line sample.

SNP rs683 genotyping. qPCR reactions were performed in duplicate in 384-well plates. cDNA ( $4.5 \mathrm{ng}$ ) was mixed with TaqMan Universal Master II - no AmpErase UNG mix with rs683 primers and specific probes supplied by Applied Biosystems. For controls, 3 ng of genotyped DNA (NA18486, NA18501, NA18917; Coriell) being homozygous (CC or AA) or heterozygous (CA) for the SNP rs683 was used per reaction.

RNA immunoprecipitation. These experiments were performed on 501Mel cells as previously published ${ }^{59}$ but with minor modifications. The $501 \mathrm{Mel}$ cells were transfected with MS2-eGFP and pMS2-3' UTR TYRP1-A for the wild type, and pMS2-lacZ for the control. Clarified cell lysates were incubated overnight at $4{ }^{\circ} \mathrm{C}$ with $4 \mu \mathrm{g}$ anti-GFP antibody (A11122, ThermoFisher Scientific). Beads (ThermoFisher Scientific) were washed three times with NT2 buffer ${ }^{60}$ and incubated with cell lysates for $2 \mathrm{~h}$. Beads were then washed five times with NT2 buffer. Pellets were resuspended in $100 \mu \mathrm{l}$ of NT2 buffer supplemented with $30 \mu \mathrm{g}$ proteinase $\mathrm{K}$ and $2 \mu \mathrm{l}$ RNAse inhibitor. RNA was eluted by heating at $55^{\circ} \mathrm{C}$ for $30 \mathrm{~min}$, purified using the mirVana miRNA Isolation Kit (Life Technologies) and quantified by TaqMan Low Density Assay (TLDA, 800 miRNAs, Applied Biosystems).

miRNA quantification by Nanostring technology. Experiments have been performed at the Curie Institute using the nCounter Human v2 miRNA Expression Assay (Nanostring) from $100 \mathrm{ng}$ of total RNA (85 metastatic melanoma). The geometric mean of the 100 most expressed miRNAs has been applied to normalize the raw data. 
Small RNA sequencing. Total RNAs were quantified using a NanoDrop 1000 Spectrophotometer (Thermo Scientific) and RNA integrity (RIN > 8) was evaluated using RNA nano-chips on the Agilent 2100 Bioanalyzer Instrument (Agilent Technologies). Small RNA libraries were generated with the NEB next small library prep set for SOLiD (New England Biolabs) and sequenced on the Applied Biosystems SOLiD 5500 wildfire system following the manufacturer's instructions. All data generated were stored on the microarray and next-generation sequencing information system Mediante ${ }^{61}$ and submitted to the GEO repository under the accession code GSE95589. Alignment was performed using Bowtie software with default parameters.

Luciferase assay. MicroRNA target validation assays were performed as described below. 501Mel cells were seeded $16 \mathrm{~h}$ before transfection at a density of 40,000 cells per well in 24-well plates. Luciferase reporter vector ( $80 \mathrm{ng}$; pMIR-REPORT) was co-transfected with 12 pmol of siRNA with Lipofectamine 2000 according to the manufacturer's instructions (ThermoFisher Scientific). Firefly luciferase activity was measured with a Promega Dual-Luciferase Reporter System using a Centro XS ${ }^{3}$ LB 960 luminometer (Berthold Technologies) $72 \mathrm{~h}$ after transfection. For MRE-16 sensors, $20 \mathrm{ng}$ of luciferase reporter vector (pLightSwitch) was co-transfected with TSB (final concentration: $30 \mathrm{nM}$ ) in 24-well plates. For the RAB17 sensor, $40 \mathrm{ng}$ was co-transfected with 16 pmol of synthetic miRNA in hepatoma cells (Huh 7.5 Drosha knockout) defective for mature hsa-miR-16 (ref. 50), seeded at 50,000 cells per well $24 \mathrm{~h}$ before transfection. For pLightSwitch vectors with individual MRE-16s, $40 \mathrm{ng}$ was reverse co-transfected with 12 pmol of synthetic miRNA in 501Mel cells seeded at a density of 60,000 cells per well. Renilla luciferase activity was measured $48 \mathrm{~h}$ after co-transfection. Luciferase activity was normalized to the protein content using a bicinchoninic acid kit (Sigma-Aldrich)

Western blot. Harvested cells were solubilized as previously described ${ }^{62}$. Membranes were probed with suitable antibodies and signals were detected using the LAS-4000 Imager (Fuji Photo Film). The primary and secondary antibodies are described in Supplementary Table 7. Anti-TYRP1 (PEP1) was kindly provided by V. J. Hearing (National Institutes of Health, Bethesda, USA). Quantifications were performed with ImageJ software.

Cell-density evaluation. Cell density was assessed using a methylene blue colorimetric assay ${ }^{62}$. Briefly, cells were fixed for at least $30 \mathrm{~min}$ in $95 \%$ ethanol. Following ethanol removal, the fixed cells were dried and stained for $30 \mathrm{~min}$ with $1 \%$ methylene blue dye in borate buffer. After four washes with tap water, $100 \mu \mathrm{l}$ of $0.1 \mathrm{~N}$ $\mathrm{HCl}$ was added to each well. Plates were next analysed with a spectrophotometer at $620 \mathrm{~nm}$.

Proliferative index evaluation. The proliferative index was evaluated for the 18 cell lines derived from metastatic melanomas ${ }^{56}$ in 96 -well plates by crystal violet assay as previously described ${ }^{63}$. On each plate, blank wells containing medium alone were used to measure the background. The proliferative index refers to the ratio of absorbance at day 3 over day 1 .

Proliferation assays. For each condition, $1.5 \times 10^{4} 501 \mathrm{Mel}$ cells or $2.5 \times 10^{4} \mathrm{ME} 1402$ cells (shCTR or shTYRP1, moderate knockdown) were plated in three $35-\mathrm{mm}$ diameter dishes and cultivated in medium with $10 \%$ fetal bovine serum (FBS). Every two days after plating, cells were counted using Malassez chambers. Calculation of mean values allowed for the plotting of growth curves.

Apoptosis assay. At the end of the treatment, cells were stained by adding $25 \mu \mathrm{l}$ of a $5 \times$ dye mixture containing Hoechst $33342\left(5 \mu \mathrm{g} \mathrm{ml}^{-1}\right)$ and YO-PRO-1 $\left(4 \mu \mathrm{g} \mathrm{m}^{-1}\right)$ directly into $100 \mu \mathrm{l}$ of culture media and incubated at $37^{\circ} \mathrm{C}$ for $30 \mathrm{~min}$. Cells were imaged, analysed and counted using an ArrayScan VTI High-Content Systems (ThermoFisher Scientific). The number of apoptotic cells (green cells) is expressed as a function of total Hoechst-positive cells.

Immunohistochemistry. Paraffin-embedded tissue was cut at $4 \mu \mathrm{m}$, mounted on positively charged slides and dried at $58^{\circ} \mathrm{C}$ for $60 \mathrm{~min}$. Staining was performed on the Ventana Discovery XT Automated IHC stainer (Roche). After deparaffinization at $75^{\circ} \mathrm{C}$ for $8 \mathrm{~min}$, slides were stained for $4 \mathrm{~min}$ with haematoxylin and rinsed. Slides were manually dehydrated and coverslipped. Samples were scanned using the scanner Nanozoomer NDP (Hamamatsu Photonics). Toxicity evaluation was blindly examined by two independent pathologists. Representative micrographs of liver and kidney from PDX mice exposed to TSB-C1 or TSB-T3 were selected according to the pathologists.

Gene expression analysis. Experiments were performed using a SurePrint G3 Human GE $8 \times 60 \mathrm{~K}$ Microarray kit (Agilent Technologies). Total RNA was amplified and labelled using a one-colour labelling protocol with the Low Input Quick
Amp Labeling kit (5190-2305; Agilent). Hybridized slides were scanned with the Agilent G2505C microarray scanner. Raw data were pre-processed and normalized with the Bioconductor LIMMA package ${ }^{64}$; the SVA package ${ }^{65}$ was used to remove batch effects from the two hybridization experiments (http://bioconductor.org) MultiExperiment Viewer (MeV v4.8) software was used for the significance analysis of microarrays (SAM), and a false discovery rate (FDR) of zero was used to identify transcripts that were differentially expressed between siRNA controls (CTR, siNT1, and siNT2) and siTYRP1 (no. 1, 2 or 3) (GEO GSE70561). Genes downregulated $(n=18)$ following siTYRP1 were compared with upregulated genes $(n=31)$ using comparison analysis (Ingenuity Pathway Analysis, IPA, Version 31813283). The activation $Z$-score assess the match of observed and predicted up/downregulation patterns, serving as both a significance measure and a predictor for the activation state of a regulator.

TCGA analyses. TYRP1 and RAB17 expression levels of 473 melanoma patients (TCGA skin cutaneous melanoma SKCM, IlluminaHiSeq) were downloaded from the UCSC cancer browser (https://genome-cancer.ucsc.edu) and are generated by the TCGA Research Network (http://cancergenome.nih.gov). NRTN mRNA and miR-16 expression data for 349 and 369 metastatic melanoma patients, respectively, were downloaded as explained below. Patients were ranked decreasingly according to TYRP1 and RAB17 expression. Three equal-sized groups were determined $(n=157$ each), containing high, intermediate and low expressers. Patients were ranked decreasingly according to NRTN or TYRP1 and NRTN expression. The highest and lower quarter groups ( $n=46$ each) were chosen for analysis. For miR-16 expression, high and low expressers were determined on the basis of median expression. Overall survival of high and low TYRP1 and RAB17 expressers, of NRTN expressers, of NRTN and TYRP1 expressers, and of miR-16 expressers was assessed using the Kaplan-Meier estimator in GraphPad Prism software.

In silico analyses. The miRNA-binding sites on TYRP1 mRNA were predicted using the web-based programs TargetScan 7.0 (ref. 4) and RNAhybrid ${ }^{34}$, both available online.

Statistics and reproducibility. Data are presented as mean \pm s.d. unless otherwise specified, and differences were considered significant at a $P$ value of less than 0.05 . In the box plots, the line within the box is the median, the bottom and top of the box are, respectively, the first and the third quartiles, and the whiskers represent the minimum and maximum of all the data points. Comparisons between groups normalized to a control were carried out by a two-tailed $t$-test with Welch's correction or one-way ANOVA followed by the Holm-Sidak's multiple comparisons test when more than two groups were compared to the same control condition. When at least two factors were compared between two groups, a two-way ANOVA (without adjustment) was used. The statistical significance between two independent groups of patient samples or treated mice was examined using the Mann-Whitney test. Statistical correlation between two continuous variables was assessed using Spearman's rho (for $n<30$ individuals) or Pearson's rho (for $n \geq 30$ individuals) test. Overall survival was estimated using the Kaplan-Meier method. Univariate analysis using the Cox regression model or log-rank test, as specified, was performed to estimate hazard ratios (HRs) and $95 \%$ confidence intervals (CIs). All statistical analyses were performed using Prism 6 software (GraphPad, La Jolla) except for Kaplan-Meier analyses of Figs 3d, 4j and Supplementary Fig. 5a,b, which used SPSS Statistics 15.0 (IBM SPSS). All experiments were performed three or more times independently under similar conditions, unless otherwise specified in the figure legends.

Data availability. Small RNA-seq and microarray data that support the findings of this study have been deposited in the Gene Expression Omnibus (GEO) under accession codes GSE95589 and GSE70561, respectively. The human melanoma data set (skin cutaneous melanoma SKCM, IlluminaHiSeq) was derived from the TCGA Research Network: http://cancergenome.nih.gov. The data set derived from this resource that supports the findings of this study is available at https://genomecancer.ucsc.edu. Source data for Figs 1-6 and Supplementary Figs 1-6 have been provided as Supplementary Table 8, except for patient raw data. Source data for Fig. $5 \mathrm{f}$ have been provided as Supplementary Table 5. All other data supporting the findings of this study are available from the corresponding author on reasonable request.

56. Verfaillie, A. et al. Decoding the regulatory landscape of melanoma reveals TEADS as regulators of the invasive cell state. Nat. Commun. 6, 6683 (2015).

57. David, S. et al. In vivo imaging of DNA lipid nanocapsules after systemic administration in a melanoma mouse model. Int. J. Pharm. 423, 108-115 (2012).

58. Bonci, D. et al. The miR-15a-miR-16-1 cluster controls prostate cancer by targeting multiple oncogenic activities. Nat. Med. 14, 1271-1277 (2008). 
59. Keene, J. D., Komisarow, J. M. \& Friedersdorf, M. B. RIP-Chip: the isolation and identification of mRNAs, microRNAs and protein components of ribonucleoprotein complexes from cell extracts. Nat. Protoc. 1, 302-307 (2006).

60. Kim, H. H. et al. HuR recruits let-7/RISC to repress c-Myc expression. Genes Dev. 23, 1743-1748 (2009).

61. Le Brigand, K. \& Barbry, P. Mediante: a web-based microarray data manager. Bioinformatics 23, 1304-1306 (2007).

62. Gilot, D. et al. RNAi-based screening identifies kinases interfering with dioxinmediated up-regulation of CYP1A1 activity. PLOS ONE 6, e18261 (2011).
63. Krayem, M. et al. Prominent role of cyclic adenosine monophosphate signalling pathway in the sensitivity of ${ }^{\mathrm{WT}} \mathrm{BRAF} /{ }^{\mathrm{WT}}$ NRAS melanoma cells to vemurafenib. Eur. J. Cancer 50, 1310-1320 (2014).

64. Smyth, G. K., Michaud, J. \& Scott, H. S. Use of within-array replicate spots for assessing differential expression in microarray experiments. Bioinformatics 21, 2067-2075 (2005)

65. Leek, J. T., Johnson, W. E., Parker, H. S., Jaffe, A. E. \& Storey, J. D. The SVA package for removing batch effects and other unwanted variation in highthroughput experiments. Bioinformatics 28, 882-883 (2012). 


\section{SUPPLEMENTARY INFORMATION}

In the format provided by the authors and unedited.

a

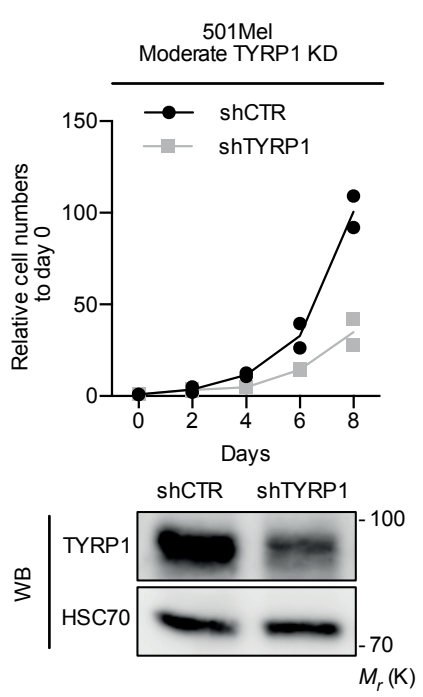

b
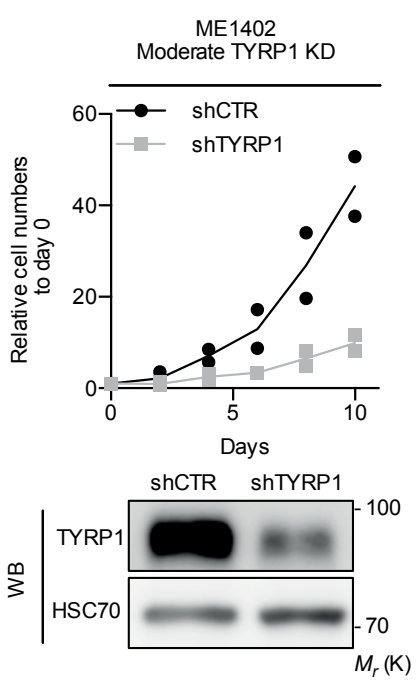

C

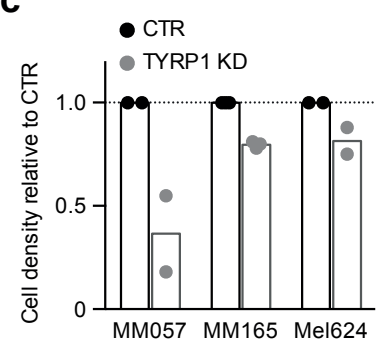

d

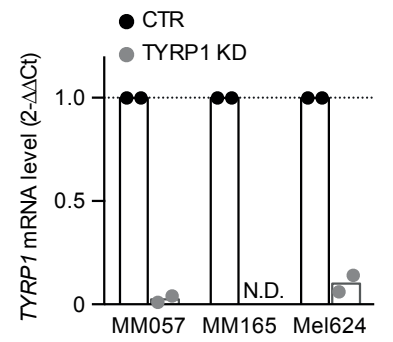

e

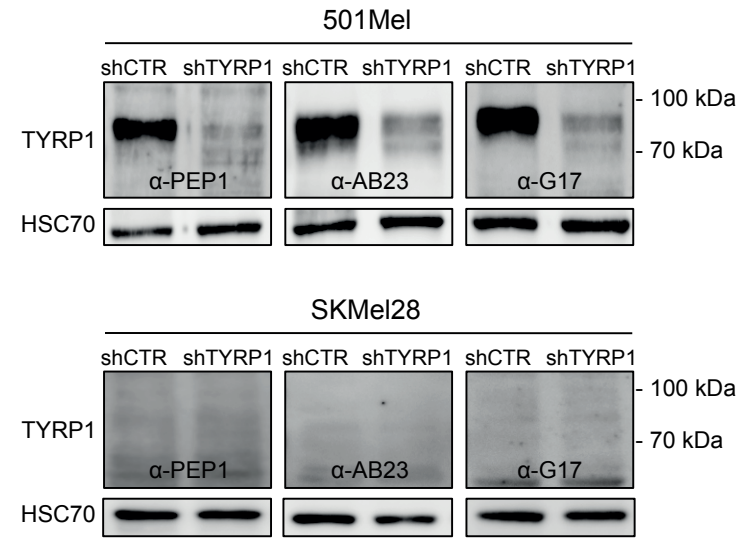

Supplementary Figure 1 Moderate TYRP1 mRNA knockdown reduces melanoma cell proliferation. (a-b) Proliferation rate of shCTR and ShTYRP1 (moderate KD) $501 \mathrm{Mel}$ (a) or ME1402 cells (b). Cells were counted every two days during eight or ten days ( $n=2$ biologically independent experiments in triplicate). TYRP1 protein was detected by Western blot experiments; pictures are representative of three independent experiments. HSC70 serves as loading control. (c-d) TYRP1 knockdown in two melanoma short-term cultures (MM057 \& MM165) and in Mel624 melanoma cell line. Cell density (c) and TYRP1 mRNA levels (d) have been evaluated 4 or 7 days after infection (shTYRP1) or transfection (siTYRP1). N.D. for not detected. Each histogram represents the mean of 2 or 3 biologically independent experiments ( $n=2$ for cell density experiments; $n=2$ for MM057 \& Mel624 and $n=3$ for MM165 for TYRP1 mRNA level quantification). (e) TYRP1 knockdown in two melanoma cell lines expressing TYRP1 mRNA. Three different antibodies (PEP1, AB23 \& G17) were used to confirm the absence of the TYRP1 protein in SKMel28-luc cells. Pictures are representative of three independent experiments. Source data are available in Supplementary Table 8 and unprocessed original blots are shown in Supplementary Fig.7. 


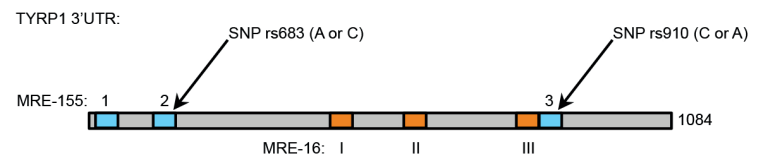

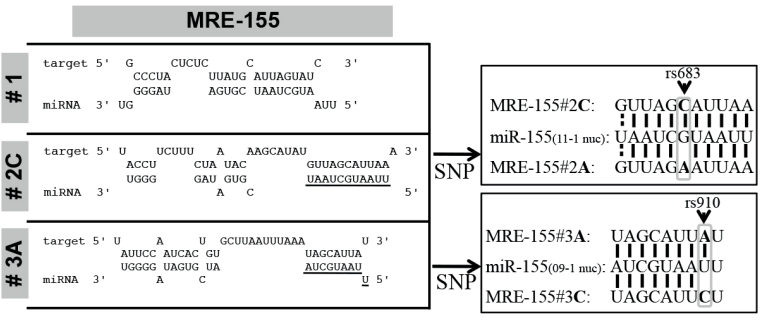

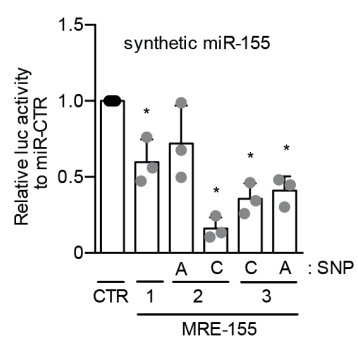

d

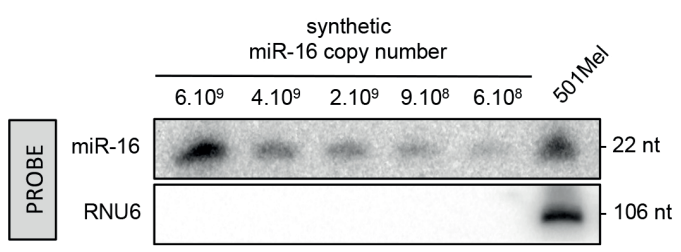

e

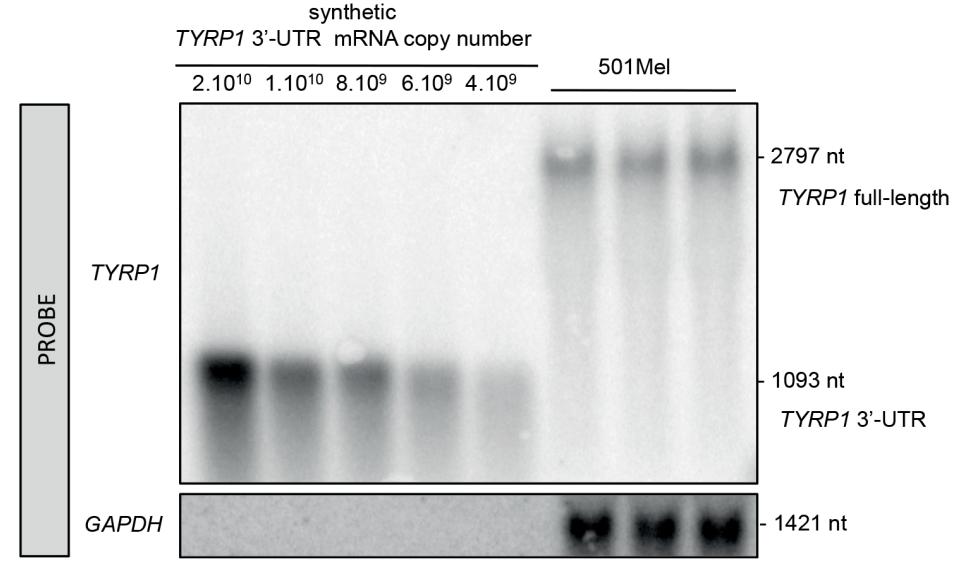

Supplementary Figure 2 miR-16 reduces TYRP 1 mRNA decay induced by miR-155. (a) Cartoon illustrating the 3'UTR of human TYRP1 with the position of the SNPs rs683 and rs910, the three MRE-155 sites (blue) and the three putative MRE-16 sites (orange). (b) MRE-155 sequences on TYRP1 3'UTR. TYRP1-C corresponds to the NM_000550.2 and TYRP1-A to NM_000550.1. Alignments have been performed using RNAhybrid ${ }^{34}$ only a few targets are known. In contrast to plant miRNAs, which usually bind nearly perfectly to their targets, animal miRNAs bind less tightly, with a few nucleotides being unbound, thus producing more complex secondary structures of miRNA/target duplexes. Here, we present a program, RNA-hybrid, that predicts multiple potential binding sites of miRNAs in large target RNAs. In general, the program finds the energetically most favorable hybridization sites of a small RNA in a large RNA. Intramolecular hybridizations, that is, base pairings between target nucleotides or between miRNA nucleotides are not allowed. For large targets, the time complexity of the algorithm is linear in the target length, allowing many long targets to be searched in a short time. Statistical significance of predicted targets is assessed with an extreme value statistics of length normalized minimum free energies, a Poisson approximation of multiple binding sites, and the calculation of effective numbers of orthologous targets in comparative studies of multiple organisms. We applied our method to the prediction of Drosophila miRNA targets in 3'UTRs and coding sequence. RNAhybrid, with its accompanying programs RNAcalibrate and RNAeffective, is available for download and as a Web tool on the Bielefeld Bioinformatics Server (http:// bibiserv.techfak.uni-bielefeld.de/rnahybrid/. Underlined sequences on MRE155\#2C and MRE-155\#3A are detailed in boxes on the right to show the position of the SNPs in the two alleles of TYRP1. Arrows indicate the SNP rs683 and rs910 positions. (c) Effects of synthetic miR-155 on the identified regions of TYRP1 MRE-155 in $501 \mathrm{Mel}$. Luciferase activity was evaluated $48 \mathrm{~h}$ after transfection. Each histogram represents the mean \pm s.d. of $n=3$ biologically independent experiments; two-sided unpaired t-test with Welch's correction; * $p<0.05$. (d) Northern blot quantification of miR-16 in $501 \mathrm{Mel}$ cells. The signal (from $501 \mathrm{Mel}$ cells) was fit to the standard curve from synthetic titration signals to give final copy number per cell. RNU6 served as a loading control. Pictures are representative of three experiments. (e) Northern blot quantification of TYRP1 mRNA in $501 \mathrm{Mel}$ cells. The signal (from $501 \mathrm{Mel}$ cells) was fit to the standard curve from the TYRP1 3'UTR's synthetic titration signal to give final copy number per cell. GAPDH serves as a loading control. Picture presents three biological replicates of $501 \mathrm{Mel}$. Source data are available in Supplementary Table 8. 


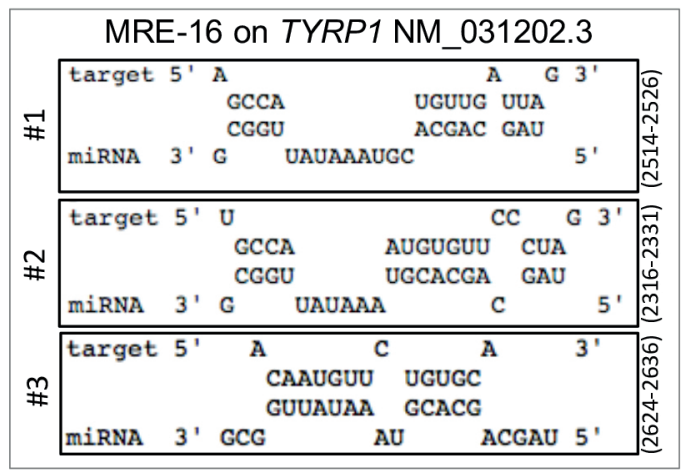

b

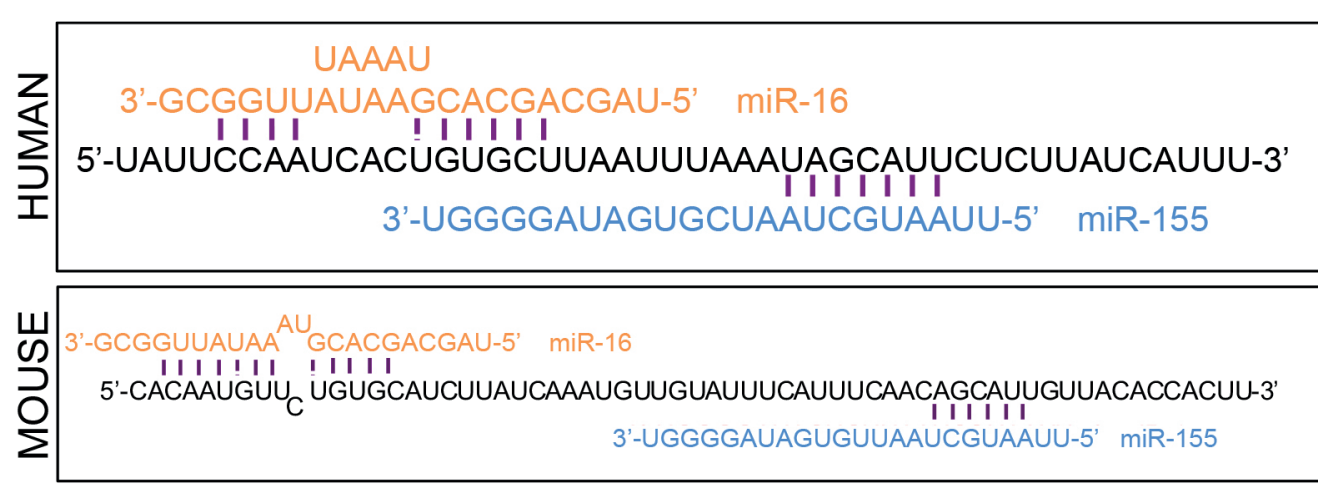

C

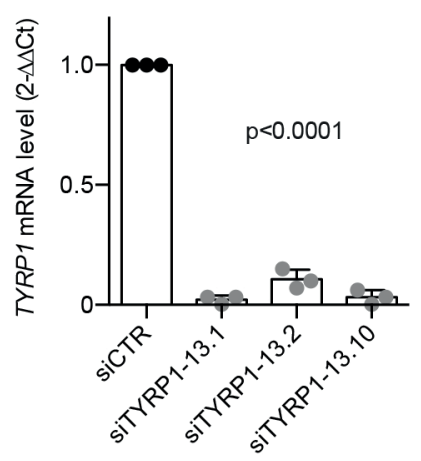

d

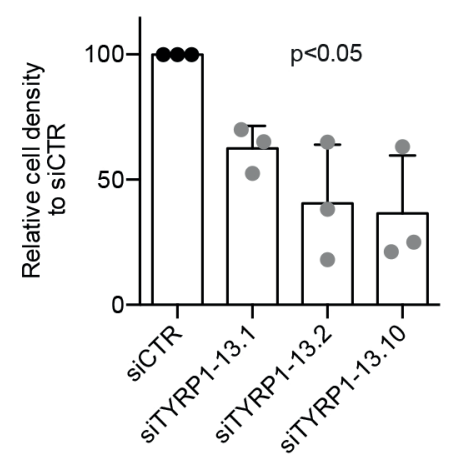

e

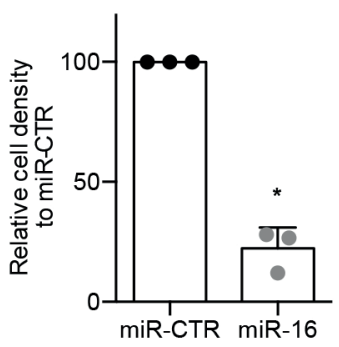

Supplementary Figure 3 MRE-16 on human and mouse TYRP1 mRNA and biological consequences. (a) MRE-16s' sequences on mouse TYRP1 3'UTR (NM_031202.3). Alignments have been performed using RNAhybrid ${ }^{34}$ only a few targets are known. In contrast to plant miRNAs, which usually bind nearly perfectly to their targets, animal miRNAs bind less tightly, with a few nucleotides being unbound, thus producing more complex secondary structures of miRNA/target duplexes. Here, we present a program, RNA-hybrid, that predicts multiple potential binding sites of miRNAs in large target RNAs. In general, the program finds the energetically most favorable hybridization sites of a small RNA in a large RNA. Intramolecular hybridizations, that is, base pairings between target nucleotides or between miRNA nucleotides are not allowed. For large targets, the time complexity of the algorithm is linear in the target length, allowing many long targets to be searched in a short time. Statistical significance of predicted targets is assessed with an extreme value statistics of length normalized minimum free energies, a Poisson approximation of multiple binding sites, and the calculation of effective numbers of orthologous targets in comparative studies of multiple organisms. We applied our method to the prediction of Drosophila miRNA targets in 3'UTRs and coding sequence. RNAhybrid, with its accompanying programs RNAcalibrate and RNAeffective, is available for download and as a Web tool on the Bielefeld Bioinformatics Server (http://bibiserv.techfak.uni-bielefeld. de/rnahybrid/. (b) Schematic representation of the interaction (purple base paring) of miR-16 (orange) and miR-155 (blue) with human and mouse TYRP1 MRE-16\#3 and MRE-155\#3 respectively. (c-d) TYRP1 knockdown in mouse B16-F10 melanoma cells using three different siRNAs. TYRP1 mRNA levels (c) and cell density (d) have been respectively evaluated at 5 and 3 days after siRNA transfection; one-way ANOVA with Holm-Sidak's multiple comparisons test. (e) Effect on cell density of synthetic miR-16 transfected in mouse B16-F10 melanoma cells 3 days after miRNA transfection; two-sided unpaired t-test with Welch's correction, ${ }^{*} p<0.05$. Each histogram represents the mean \pm s.d. ( $n=3$ biologically independent experiments). Source data are available in Supplementary Table 8. 
a
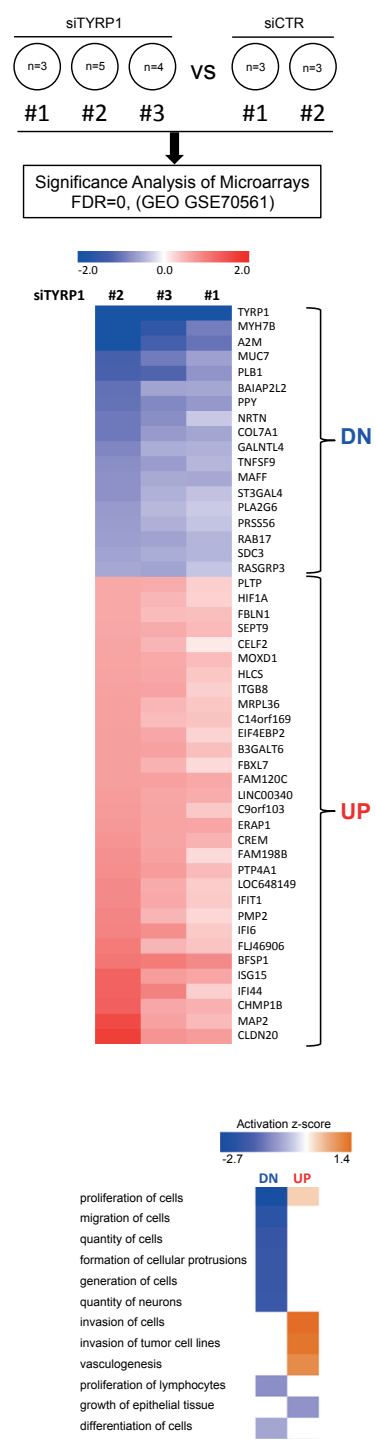

Supplementary Figure 4 TYRP1 silencing decreases expression level of several mRNAs. (a) Workflow to identify deregulated RNAs in TYRP1 KD cells. Gene expression profile of cells transfected with three different siRNAs targeting TYRP1 or siRNA CTR. Significance analysis of microarrays was done as described in methods. siRNA efficacy for TYRP1 KD is \#2>\#3>\#1. Heatmap focused on deregulated RNAs in function of siTYRP1 efficacy (top) and a z-score has been calculated using Ingenuity Pathway Analysis (IPA) (bottom). (b) mRNA expression levels of TYRP1, MAFF, NRTN, RAB17 and RasGRP3 in response to TYRP1 KD using three different siRNAs (\#1-3) targeting the ORF of TYRP1 in $501 \mathrm{Mel}$ cells $(n=4$ biologically independent experiments except for RASGRP3 expression measurement which results from $n=3$ biologically independent experiments). (c) mRNA expression levels of TYRP1, MAFF, NRTN, RAB17 and RasGRP3 in response to TYRP1 KD using shTYRP1 targeting the ORF of TYRP1 in SKMel28-luc cells ( $n=3$ biologically independent experiments). (d) Cell density of $501 \mathrm{Mel}$ cells in response to siRNAs targeting MAFF, NRTN, RAB17 or RasGRP3 ( $n=3$ biologically independent experiments; one-way ANOVA with Holm-Sidak's multiple comparisons test, ${ }^{*} p<0.05$ ). Two siRNAs were used by target. (e) mRNA expression levels in response to synthetic miR-16. Each histogram represents the mean \pm s.d. ( $n=3$ biologically independent experiments). TYRPI and $R A B 17$ mRNA expression in response to synthetic miR-16 are reported in Fig. $2 \mathrm{~d}$ and $4 \mathrm{~b}$, respectively. (f) The MRE-16s' sequence on human MAFF and b
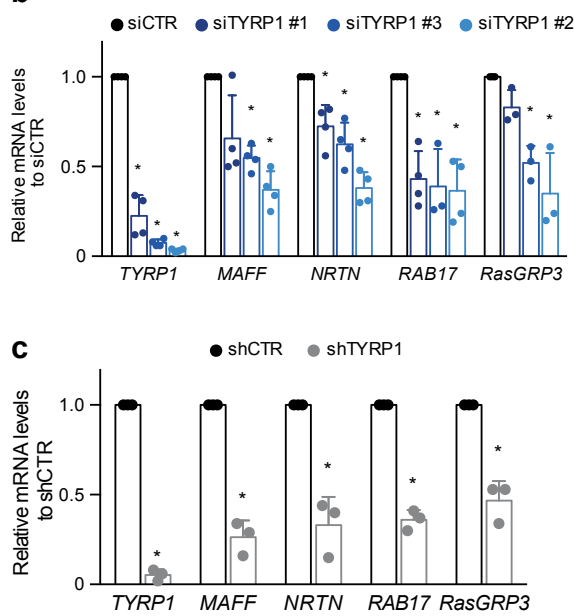

d
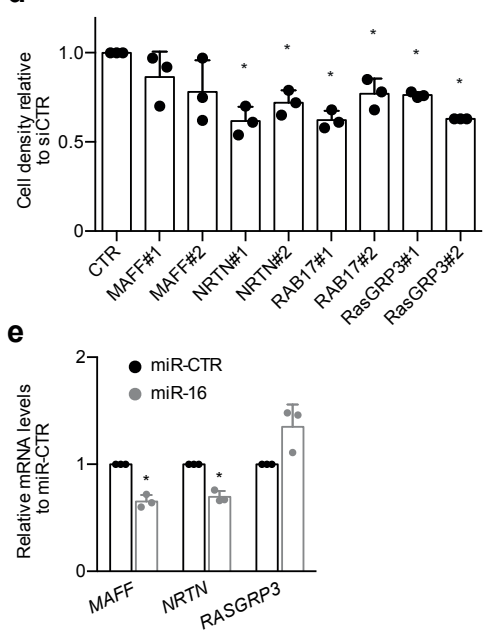

f

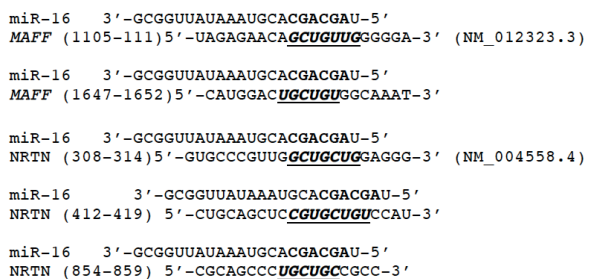

miR-16 3'-GCGGUUAUAAAUGCACGACGAU-5' MAFF (1105-111)5'-UAGAGAACAGCUGUUGGGGGA-3' (NM 012323.3) miR-16 3'-GCGGUUAUAAAUGCACGACGAU-5 MAFF (1647-1652)5'-CAUGGACUGCUGUGGCAAAT-3 MiR-16 3'-GCGGUUAUAAAUGCACGACGAU-5' NRTN (308-314) 5'-GUGCCCGUUGGCUGCUGGAGGG-3' (NM_004558.4) miR-16 3'-GCGGUUAUAAAUGCACGACGAU- 5 ' NRTN (412-419) 5'-CUGCAGCUCCGUGCUGUCCAU-3' miR-16 3'-GCGGUUAUAAAUGCACGACGAU-5'

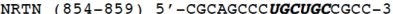

NRTN mRNAs have been identified using RNAhybrid ${ }^{34}$ only a few targets are known. In contrast to plant miRNAs, which usually bind nearly perfectly to their targets, animal miRNAs bind less tightly, with a few nucleotides being unbound, thus producing more complex secondary structures of miRNA/ target duplexes. Here, we present a program, RNA-hybrid, that predicts multiple potential binding sites of miRNAs in large target RNAs. In general, the program finds the energetically most favorable hybridization sites of a small RNA in a large RNA. Intramolecular hybridizations, that is, base pairings between target nucleotides or between miRNA nucleotides are not allowed. For large targets, the time complexity of the algorithm is linear in the target length, allowing many long targets to be searched in a short time. Statistical significance of predicted targets is assessed with an extreme value statistics of length normalized minimum free energies, a Poisson approximation of multiple binding sites, and the calculation of effective numbers of orthologous targets in comparative studies of multiple organisms. We applied our method to the prediction of Drosophila miRNA targets in 3'UTRs and coding sequence. RNAhybrid, with its accompanying programs RNAcalibrate and RNAeffective, is available for download and as a Web tool on the Bielefeld Bioinformatics Server (http://bibiserv.techfak.uni-bielefeld.de/rnahybrid/. For $\mathbf{b}$-c and e, two-sided unpaired t-tests with Welch's correction were done $\left({ }^{*} p<0.05\right)$. For $\mathbf{b}-\mathbf{e}$, values correspond to the mean \pm s.d. Source data are available in Supplementary Table 8. 
a

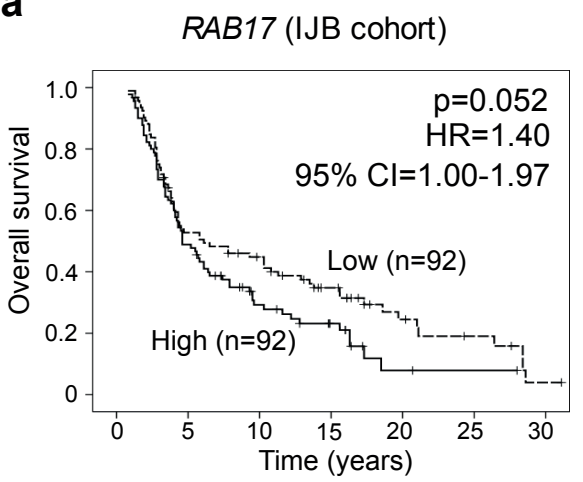

d

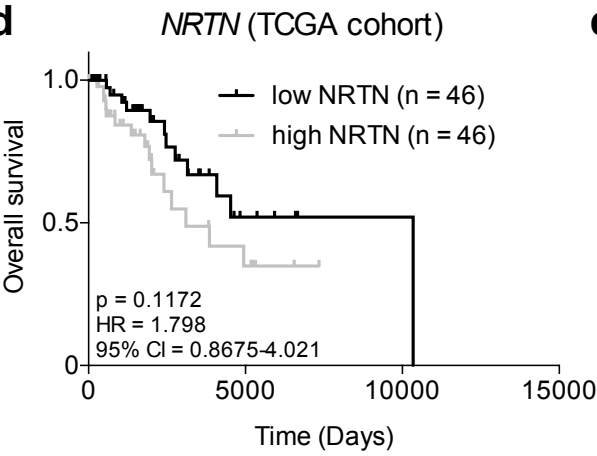

f

miR-16 (IJB cohort)

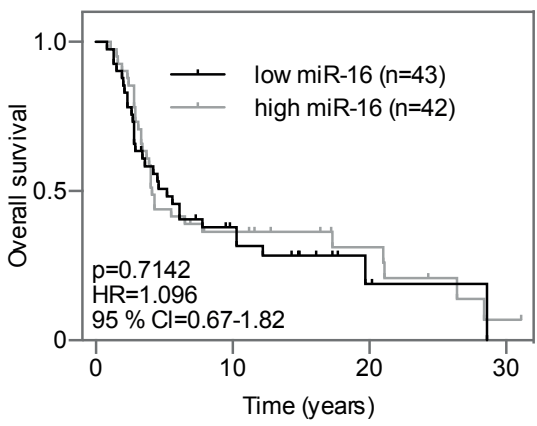

b

TYRP1 + RAB17 (IJB cohort)
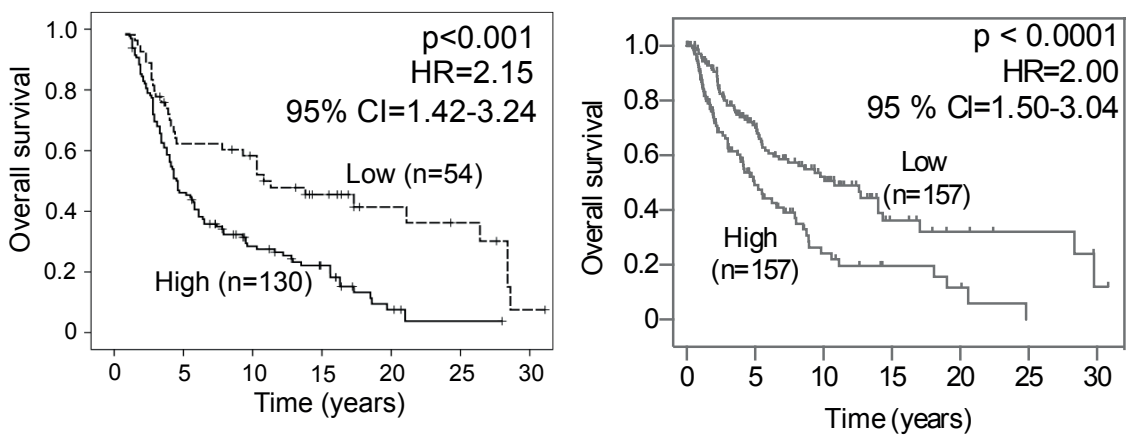

e

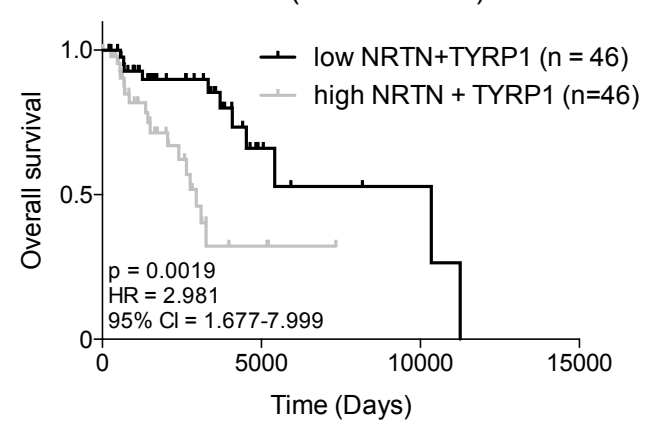

g

miR-16 (TCGA cohort)

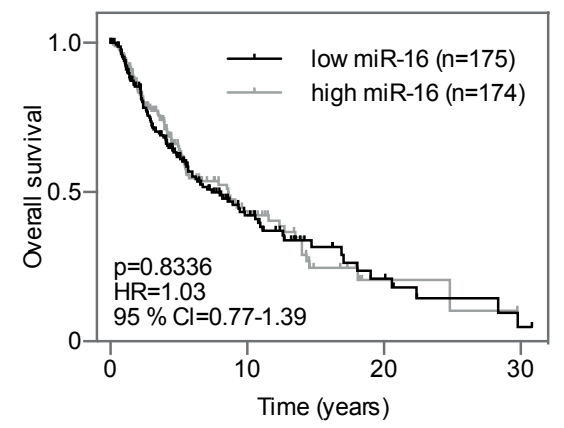

Supplementary Figure 5 Overall survival of patients with metastatic melanoma according to TYRP1, RAB17, NRTN mRNAs or miR-16 expression levels. (a-b) Determination of overall survival (OS) curves by Kaplan-Meier analysis, according to the expression levels of $R A B 17$ (a) or TYRP1 and RAB17 (b). Based on 184 skin and lymph node metastases from melanoma patients (IJB cohort). High and low expression of TYRPI and $R A B 17$ (a) alone were defined based on their median values and scored as 1 and 0 , respectively. For combination (b), scores of TYRP1 (1 or 0 ) and $R A B 17$ ( 1 or 0 ) are added and the resulting scores 1 and 2 are combined as the high score ( $n=130$ patients), which was significantly different from the low score 0 ( $n=54$ patients) regarding to OS values (two-sided Mann-Whitney test, $\mathrm{p}=0.004$ ). Cox regression was used to calculate $P$ values, hazard ratios (HR) and $95 \%$ confidence intervals (CI). (c) Association of TYRP1 and RAB17 expression with patient survival. TYRP1 and $R A B 17$ expression levels were assessed in the TCGA SKCM melanoma cohort. Patients were ranked decreasingly according to TYRP1 and RAB17 expression, resulting in almost three equal groups. The Kaplan-Meier curve representing the highest third of TYRP1 and RAB17 expressers shows a significantly lower OS as compared to the lowest third (log-rank test). (d-e) Determination of OS curves by Kaplan-Meier analysis, according to the expression levels of NRTN (d) or TYRP1 and NRTN (e). Patients were ranked decreasingly according to NRTN (d) or TYRP1 and NRTN expression (e), resulting in almost four equal groups. The Kaplan-Meier curve represents the highest and the lower quarters of expressers (log-rank test). (f-g) Determination of OS curves by Kaplan-Meier analysis, according to the expression levels of miR-16 from $n=85$ patients of the IJB cohort ( $f$ ) or from $n=349$ patients from the TCGA cohort (g). High and low expression groups of miR-16 were defined based on its median value (log-rank test). 
a

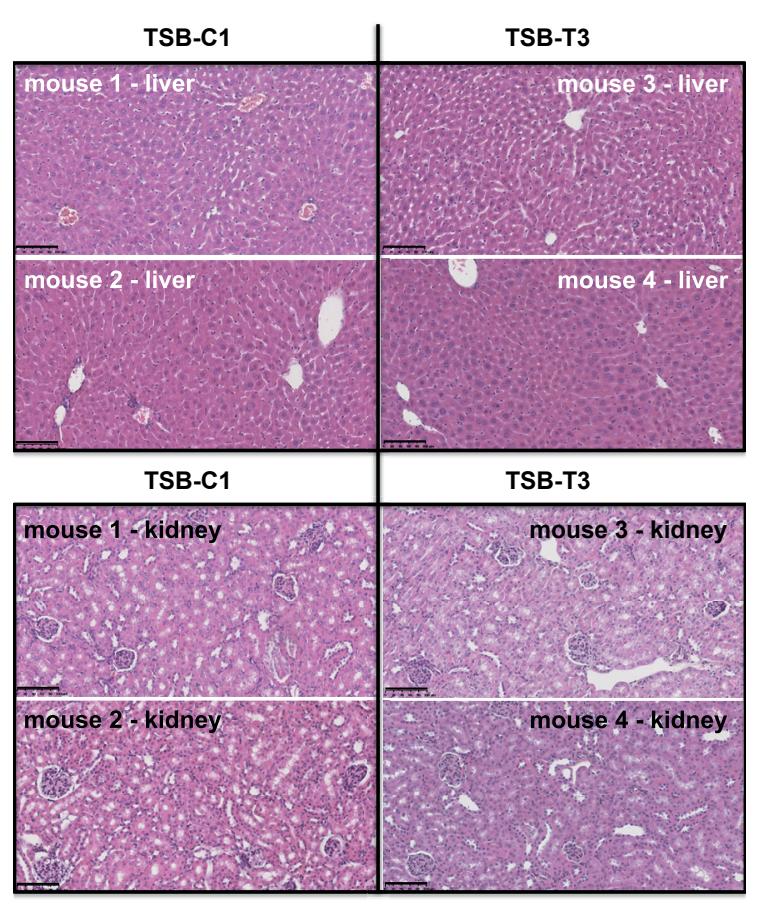

b
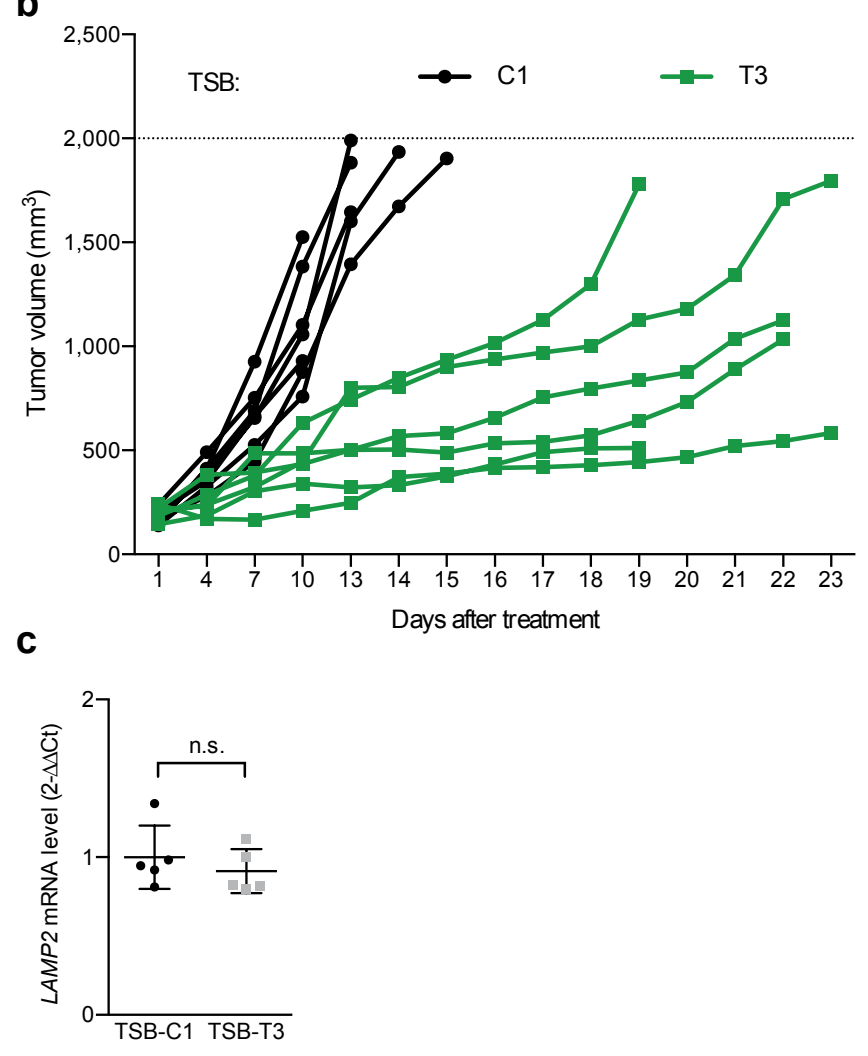

Supplementary Figure 6 Histological analyses of liver and kidney from PDX mice and long-term tumor growth. (a) Representative micrographs of liver and kidney slices stained with hematoxylin from PDX-mice exposed to TSBC1 or TSB-T3. Toxicity evaluation was blindly examined by two independent pathologists. Two mice have been showed per group among five mice analysed given similar results. Scale bar : $100 \mu \mathrm{m}$. (b) Tumor volume for individual PDX mice treated with TSB-C1 or TSB-T3 as described in Fig. 6b. (c) Quantification of $\angle A M P 2$ mRNA in melanoma tumors treated with TSB-C1 or TSB-T3 ( $n=5$ mice per group). LAMP2 is not a miR-16 target. For $\mathbf{c}$, lines represent the mean \pm s.d.; values represent fold change relative to the mean of TSB-C1 condition; two-sided unpaired $t$-tests with Welch's correction; n.S., non-significant. Source data are available in Supplementary Table 8. 

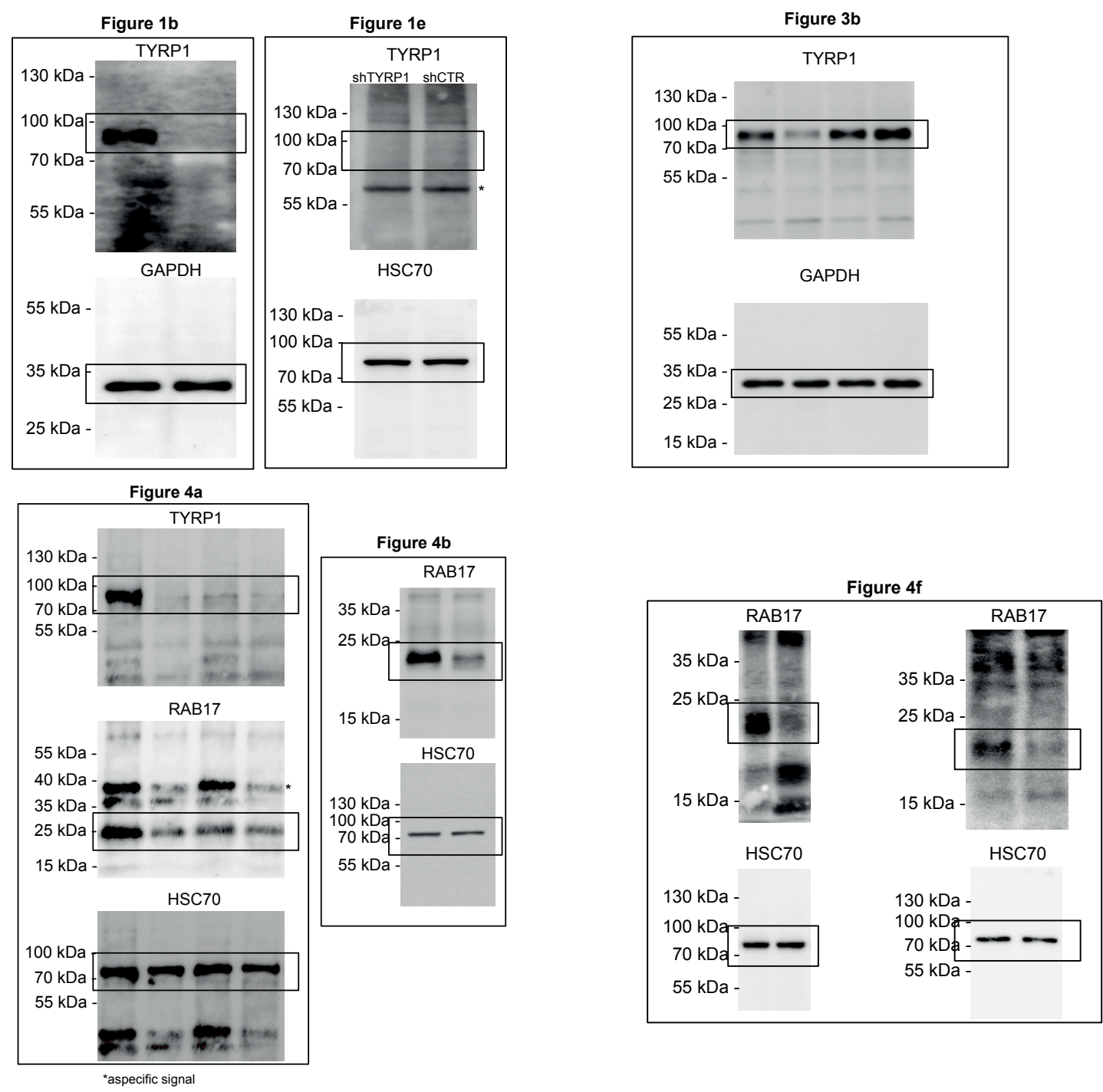

Sup. Figure 1e

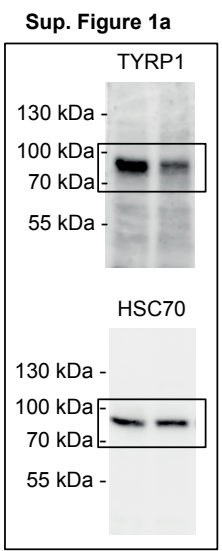

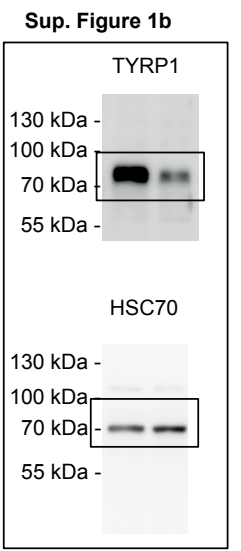

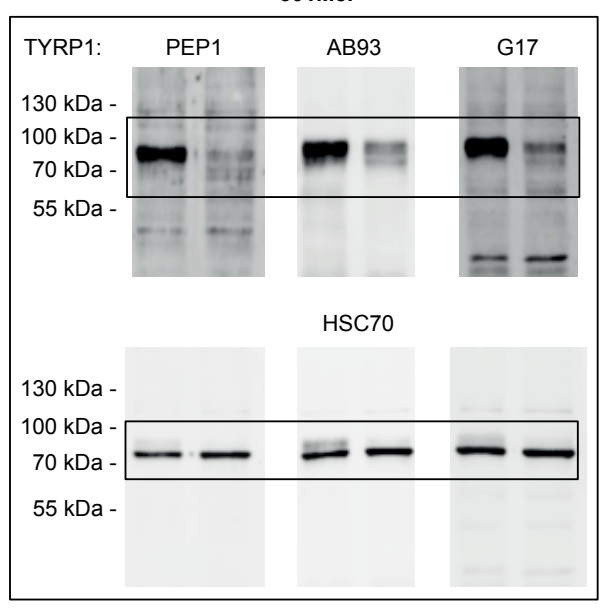

Sup. Figure 1e

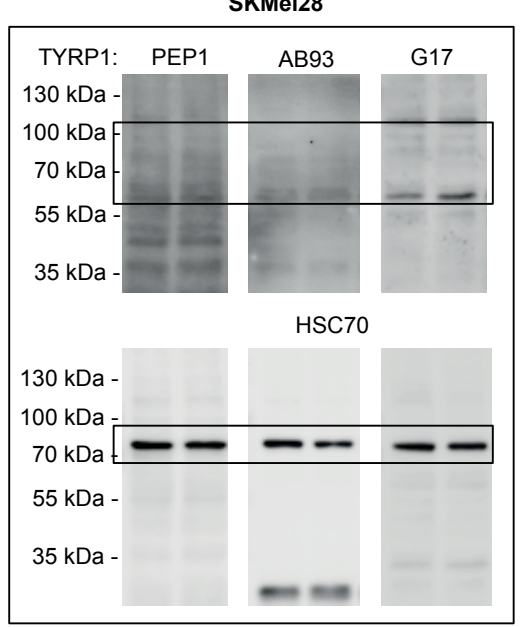

Supplementary Figure 7 Unprocessed original scans of blots. 


\section{Supplementary Table Legends}

Supplementary Table 1 Metastatic melanoma cell lines and short-term cultures.

Supplementary Table 2 miRNA quantification in 501 Mel cells.

Supplementary Table 3 Clinical characteristics of the patients and tissues from the Institute Jules Bordet cohort.

Supplementary Table 4 Correlation between TYRP1 and RAB17, NRTN or MAFF in the TCGA cutaneous skin cancer cohort.

Supplementary Table 5 TSB-T3 efficacy, mutation state and mRNAs expression in 501Mel and short-term cultures.

Supplementary Table 6 siRNA, miRNA, TSB, inserts and probes sequences.

Supplementary Table 7 Antibodies.

Supplementary Table 8 Statistics Source Data. 